\title{
Ultra-thin silica films on metals: The long and winding road to understanding the atomic structure
}

\author{
Shamil Shaikhutdinov* and Hans-Joachim Freund \\ Abteilung Chemische Physik, Fritz-Haber-Institut der Max-Planck-Gesellschaft, \\ Faradayweg 4-6, 14195 Berlin, Germany
}

\begin{abstract}
This paper critically reviews the experimental and theoretical studies on the growth of ultra-thin silica films onto metal single crystal substrates reported to date. The silica films on $\operatorname{Mo}(112)$ and $\mathrm{Ru}(0001)$ are discussed in more detail to demonstrate the key roles of the multitechnique approach and interplay between experiment and theory in the quest for understanding the atomic structure of the films. The results show the structural complexity and diversity of silica overlayers on metals, providing further information towards our understanding of the atomic structure, structural dynamics and physical and chemical properties of silica and related materials.
\end{abstract}

*Corresponding author: shaikhutdinov@fhi-berlin.mpg.de 


\section{About the Authors:}

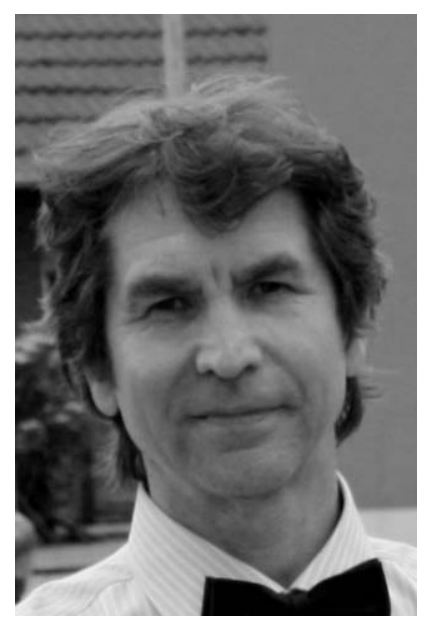

Shamil Shaikhutdinov received his PhD (1986) in Physics at the Moscow Institute of Physics and Technology studying the microwave properties of bio-organic materials and their models. Then he joined the Boreskov Institute of Catalysis at Novosibirsk to carry out surface science studies of catalytic systems. From 1994, he worked as a postdoctoral fellow in several research centres in Germany and France. Since 2004 he has been a group leader at the Department of Chemical Physics of the Fritz-Haber Institute in Berlin. His current research is focused on an understanding of structure-reactivity relationships in heterogeneous catalysis.

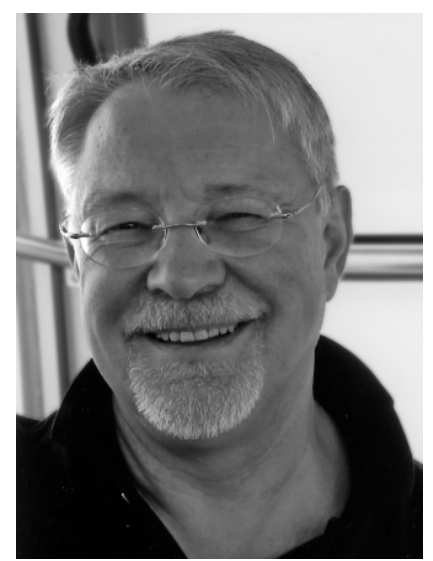

Hans-Joachim Freund studied physics and chemistry at the University of Cologne where he received his Ph.D. in 1978 and his habilitation in 1983. Between 1979 and 1981, he worked in the Physics Department at the University of Pennsylvania as a postdoctoral fellow. In 1983, he became Associate Professor at Erlangen University and in 1987 Professor at Bochum University, and in 1995, he accepted a position as scientific member and director of the Department of Chemical Physics at the Fritz-HaberInstitut der Max-Planck-Gesellschaft in Berlin. He serves as Honorary Professor of five universities. He received several national and international awards and he is a member of several academies, scientific societies, and advisory boards of scientific journals. 


\section{Introduction}

Silicon dioxide $\left(\mathrm{SiO}_{2}\right)$ is one of the most abundant materials on our planet and is the key material in many modern technological applications. It has been widely studied because of its importance to geology, material science, microelectronics, photonics, and catalysis.

There is a long-standing interest in the synthesis of thin $\mathrm{SiO}_{2}$ films for microelectronic processing. The properties of silica layers are essential for the performance of electronic devices. The understanding of ultra-thin silica films becomes even more important for the new generation of metal-oxide-semiconductor transistors, which use silica films as the gate dielectric. Further miniaturization critically depends on our knowledge about the transition region of substoichiometric $\mathrm{SiO}_{\mathrm{x}}$ at the $\mathrm{SiO}_{2} / \mathrm{Si}$ interface. ${ }^{[1]}$

Silica (together with alumina) is also a widely used support in catalysis. It is evident that the performance of silica in heterogeneous catalysts is primarily determined by surface species, such as silanols ( $\mathrm{Si}-\mathrm{OH})$, regardless whether silica is involved either as a support for catalyst particles or as a reactive surface. ${ }^{[2]}$ The surface structures become very complex in porous systems like zeolites. The current understanding of the relation between structure and chemical reactivity of these complex materials often comes from studies employing bulk-sensitive techniques and from theoretical calculations based on a general wisdom about their structure. The application of surface-sensitive techniques to elucidate the atomic structure and the mechanism of catalytic reactions on silica-based materials remains challenging.

Finally, vitreous silica is the basis of traditional silicate glasses. Despite about a hundred years of studies in this field, the greatest surprise about glasses is just how little is really known, as opposed to merely postulated, about its structure. ${ }^{[3]}$ Surface-science studies of vitreous silica are rather in an "embryonic" state. 
The rational approach to tackle some of the above-mentioned fundamental issues about silica at low dimensions requires the preparation of a system which (i) is well suited to a whole "army" of the experimental and computational methods available in surface science and (ii) features atomic structures of the bulk counterparts. This can be realized in practice through the preparation of a thin film with a well-defined structure supported by a planar metal single crystal substrate. Although such an approach is currently applied to many transition metal oxides, to the best of our knowledge, there is no comprehensive review about silica and related materials.

Here, we attempt to review the experimental, often theoretically supported, studies on ultra-thin silica films grown on metals available to date. The review is organized as follows. We first start with the introduction of the basic silica polymorphs and key observations for silica thin films grown on Si single crystals, which may be considered as a benchmark to the thin silica films on metals - the main topic of this paper. The results for a silica/Mo(112) system will be discussed in more detail in order to demonstrate, on one hand, the necessity of the multitechnique approach and, on the other hand, the key role of the interplay between experiment and theory, in the quest for understanding the atomic structures. Further studies showed that the film structure strongly depends on the metal substrate used (Mo, Ni, Pd, Ru, Pt). In particular, studies on $\mathrm{Ru}(0001)$ and $\mathrm{Pt}(111)$ led to the observation of well-defined "bilayer" films only weakly bonded to the metal support, like a free-standing $\mathrm{SiO}_{2}$ sheet. In addition, these systems made possible the preparation and atomic imaging of vitreous silica in the two-dimensional form, thus opening an avenue for further studies of long standing problems of real space imaging of crystalto-glass transitions. The paper ends with some concluding remarks.

\section{Background}




\subsection{Silica polymorphs}

Silica has relatively simple crystal structures, all made up from $\mathrm{SiO}_{4}$ tetrahedra, but a rich phase diagram as depicted in Fig. 1. At atmospheric pressure, the equilibrium sequence of phase transitions, prior to melting at $\sim 1705^{\circ} \mathrm{C}$, includes $\alpha$-quartz to $\beta$-quartz (via an incommensurate phase at $\left.573^{\circ} \mathrm{C}\right)$, then to tridymite $\left(870^{\circ} \mathrm{C}\right)$, and finally to $\beta$-cristobalite $\left(1470^{\circ} \mathrm{C}\right)$. Tridymite is notable for the surprisingly high number of phases which have been reported to exist. ${ }^{[4]}$ All crystalline phases consist of infinite frameworks of corner-sharing [ $\left.\mathrm{SiO}_{4}\right]$ tetrahedra. For example, the high-temperature phases HP-tridymite and $\beta$-cristobalite consist of identical sheets of corner-sharing $\mathrm{SiO}_{4}$ tetrahedra (shown in the inset in Fig. 1) joined in a hexagonal closepacked or face cubic-centered arrangement, respectively. ${ }^{[5]}$
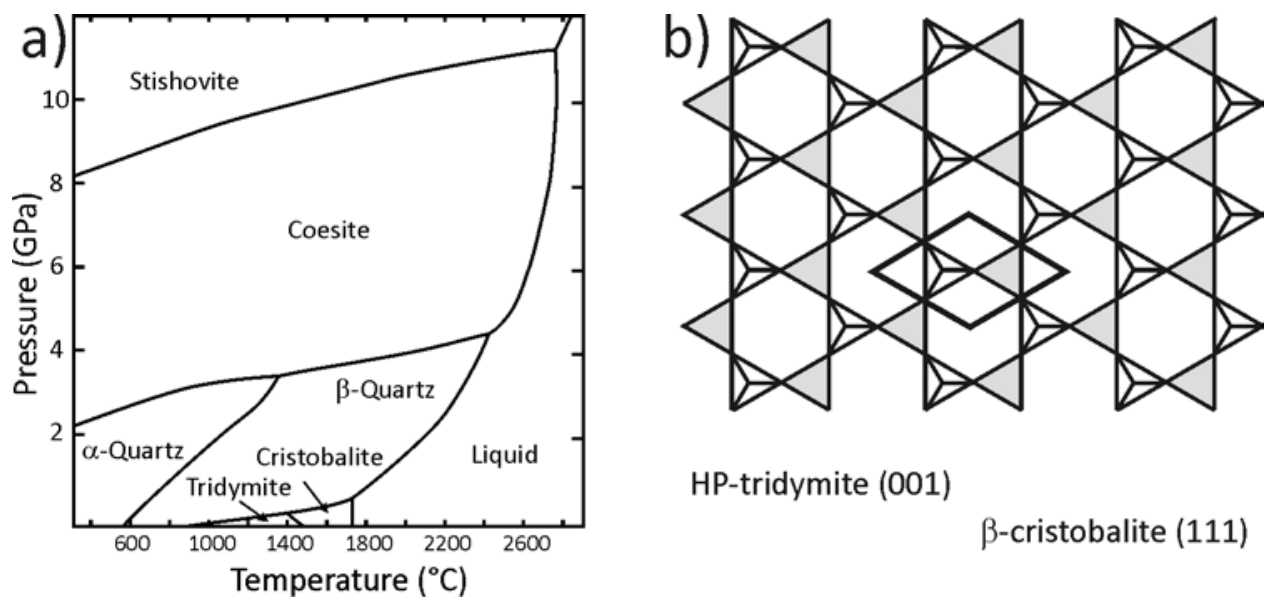

HP-tridymite (001)

$\beta$-cristobalite (111)

Figure 1. (a) Phase diagram of the $\mathrm{SiO}_{2}$ polymorphs. (b) Layers containing 6-membered rings of corner-sharing $\left[\mathrm{SiO}_{4}\right]$ tetrahedra corresponding to the HP-tridymite(001) and $\beta$-cristobalite(111) planes. The unit cell is indicated. 


\subsection{Silica thin films on silicon single crystals}

When one aims solely at the preparation of silica films (i.e., on any substrate), thermal growth on Si single crystal surfaces is the obvious choice to try first. Motivated by the key role of the $\mathrm{SiO}_{2} / \mathrm{Si}$ interface in microelectronics, oxidation of silicon single crystals was studied in a huge number of publications addressing the mechanism and kinetics of the oxide film growth, structural and electronic properties of silicon dioxide, the formation of silicon suboxides, etc (see, for example, refs. ${ }^{[6]}$ ) It is fair to say that almost every newly developed surface science technique was applied to the $\mathrm{SiO}_{2} / \mathrm{Si}$ system. Below, only a brief survey of such studies will be presented.

Auger electron spectroscopy (AES) and electron energy loss spectroscopy (EELS) studies $^{[7]}$ of the initial stages of oxidation of $\mathrm{Si}(111)-(7 \times 7)$ under well-controlled conditions for thin $(0-3 \mathrm{~nm})$ silica layers fit fairly well to a phenomenological model based on oxygen diffusion through the oxide layer under the presence of a surface electric field. ${ }^{[8]}$ Oxygen adsorption on $\mathrm{Si}(111)-(7 \times 7)$ and $\operatorname{Si}(100)-(2 \times 1)$ surfaces in the entire range of exposures from 0.1 Langmuir $\left(1 \mathrm{~L}=10^{-6}\right.$ Torr $\left.\times \mathrm{sec}\right)$ to oxidation in air in the temperature range between $100 \mathrm{~K}$ and $700 \mathrm{~K}$ was examined by high resolution EELS (HREELS). ${ }^{[9]}$ The formation of $\mathrm{SiO}_{2}$ was observed only after annealing to $700 \mathrm{~K}$, at least; at room temperature, even exposures as high as $\sim 10^{11} \mathrm{~L}$ did not produce the spectrum of $\mathrm{SiO}_{2}$.

While it is generally accepted that the bulk of silica films grown on Si crystals have the structure of vitreous (amorphous) silica (see Fig.2), the structure and properties of the interface

between $\mathrm{SiO}_{2}$ and crystalline $\mathrm{Si}$ remains controversial. A comparative HREELS study ${ }^{[10]}$ of 
quartz(0001) and silica films on $\mathrm{Si}(100)$ wafers as a function of oxide thickness indicated the presence of a transition layer of $\sim 2.5 \mathrm{~nm}$ at the $\mathrm{SiO}_{2} / \mathrm{Si}(100)$ interface. With decreasing oxide thickness, peak positions gradually shifted to lower frequencies and intensities decreased. The dielectric theory applied to a thin homogeneous supported film could not account for the observed frequency shift.

X-ray photoelectron spectroscopy (XPS) studies of differently prepared samples also

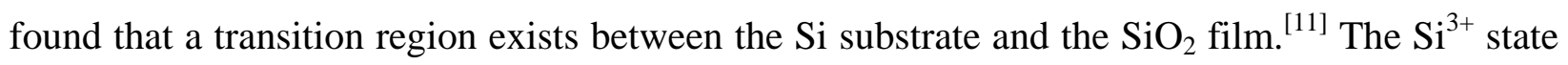
may be found up to $\sim 3 \mathrm{~nm}$ into the bulk $\mathrm{SiO}_{2}$, whereas the $\mathrm{Si}^{+}$and $\mathrm{Si}^{2+}$ states were mostly localized at the abrupt $\mathrm{SiO}_{2} / \mathrm{Si}$ interface. Interestingly, $\mathrm{Si}^{+}$species dominated at the $\mathrm{SiO}_{2} / \mathrm{Si}(111)$ interface, whereas $\mathrm{Si}^{2+}$ dominated at the $\mathrm{SiO}_{2} / \mathrm{Si}(100)$ interface. ${ }^{[12]}$ XPS studies with synchrotron radiation showed that the transition region from crystalline $\mathrm{Si}$ to amorphous $\mathrm{SiO}_{2}$ is less than 0.5 nm thick. ${ }^{[13]}$ The oxide films grown by ozone as an oxidation agent are thought to have a thinner transition layer than that of a thermally grown oxide under the same pressure and temperature. ${ }^{[14]}$ For a comprehensive analysis of XPS studies on $\mathrm{SiO}_{2} / \mathrm{Si}$ the readers are referred to a recent review by Hirose et al. ${ }^{[6 b]}$

It should be mentioned that few studies reported ordering at $\mathrm{SiO}_{2} / \mathrm{Si}(100)$ interfaces when $\mathrm{SiO}_{2}$ films were grown on passivated, ordered $(1 \times 1)$-Si(100) surfaces produced by a novel wet chemical cleaning. ${ }^{[15]}$ Ion beam channeling and nuclear resonance analysis results revealed registry of Si atoms in silica relative to the Si substrate, which was attributed to a $\beta$-cristobalite epitaxial phase. This structurally ordered phase transforms into an amorphous structure approximately $2 \mathrm{~nm}$ from the interface. Beyond this thickness, the oxide becomes amorphous. 


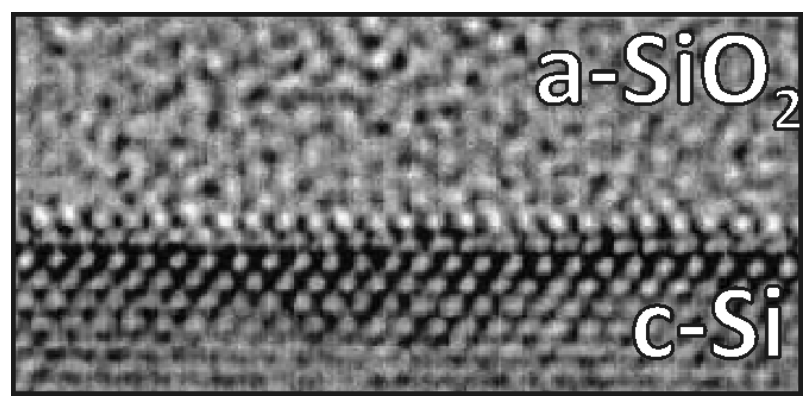

Figure 2. Cross-sectional transmission electron microscopy imaging of the interfacial $\mathrm{SiO}_{2} / \mathrm{Si}$ region. Reprinted from ref. ${ }^{[16]}$ Copyright (2001) with permission from Elsevier.

The infrared (IR) spectra of silica films are commonly analyzed in terms of their transverse-optic (TO) and longitudinal-optic (LO) vibrational modes. To fit the experimental results obtained on the $10-100 \mathrm{~nm}$ thick $\mathrm{SiO}_{2} / \mathrm{Si}$ films, disorder-induced mechanical coupling between the asymmetric stretch $\left(\mathrm{AS}_{1}\right)$ TO mode (in-phase motion of adjacent oxygen atoms) and the relatively optically inactive oxygen asymmetric stretch $\left(\mathrm{AS}_{2}\right)$ mode (out-of-phase motion of adjacent oxygen atoms) was included in the oscillator model. ${ }^{[17]}$ Coupled $\mathrm{AS}_{1}$ - and $\mathrm{AS}_{2}$-mode LO-TO frequency pairs were observed as peaks at approximately $1256-1076 \mathrm{~cm}^{-1}$ and $1160-$ $1200 \mathrm{~cm}^{-1}$, respectively. LO-TO splitting can be observed experimentally by making use of the Berreman effect. ${ }^{[18]}$ The TO modes are optically active, whereas the LO modes are optically inactive for an infinite crystal and hence cannot be observed in conventional transmission IR spectra. However, they can be detected in p-polarized oblique-incidence absorption spectra measured for thin films with thickness much smaller than the wavelength of the lattice vibration. Then the vibrational modes normal to the surface have the frequencies of the LO modes, whereas the vibrational modes parallel to the surface correspond to the TO modes. 

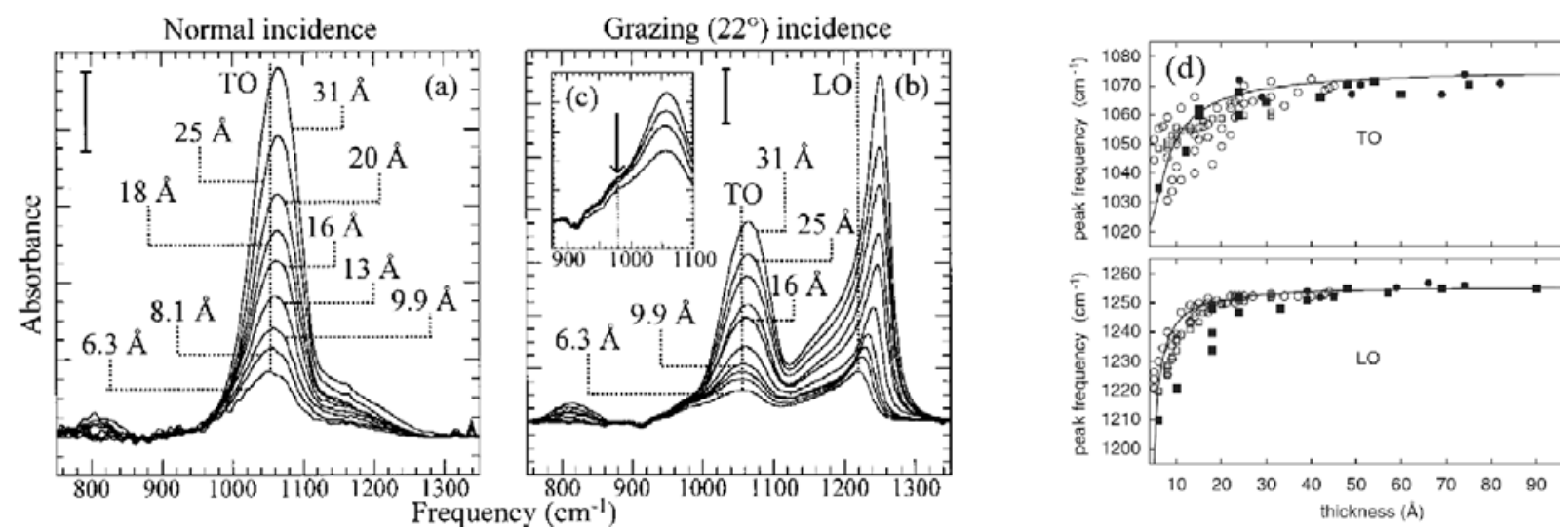

Figure 3. (a,b) IR spectra of thermally grown $\mathrm{SiO}_{2}$ thinned from 3.1 to $0.63 \mathrm{~nm}$, acquired with the incident IR beam normal (a) and grazing (b) to the sample surface. Grazing incidence spectra of the four thinnest films are expanded vertically in inset (c). Reprinted with permission from ref. $^{[19]}$. Copyright (2000) by the American Institute of Physics; (d) Position of the high-frequency TO and LO peaks as a function of oxide thickness on Si(100). The predicted theoretical frequencies, shown as a solid line, were rescaled to match the experimental data of thick $\mathrm{SiO}_{2}$ films. Reprinted with permission from ref. ${ }^{[20]}$ Copyright (2005) by the American Physical Society.

This is illustrated in Figures 3(a-c) which show the IR spectra at normal and grazing incidence of uniformly thinned, thermally grown silica films from $3 \mathrm{~nm}$ down to $0.6 \mathrm{~nm}$ (the film thickness was estimated by XPS). ${ }^{[19,21]}$ A comprehensive analysis of the spectral changes observed as a function of thickness suggested that neither image charge effects, increased stress, nor density near the interface and surface/interface roughness are not the dominant factors influencing the IR spectra. Instead, the data are best fitted by introducing a substantial component of sub-stoichiometric oxide that is prevalent within $6 \AA$ of the interface, ultimately resulting in 
the red-shift of both TO and LO modes for the thinnest films. This conclusion was theoretically supported using a scheme for calculating both the TO and LO spectra of surfaces and interfaces from first principles. ${ }^{[20]}$ Applied to the previously suggested structural model of a $\mathrm{SiO}_{2}$ film on $\operatorname{Si}(100),{ }^{[22]}$ this scheme yielded IR spectra which basically reproduced the experimentally observed shifts as shown in Fig. 3d. The effect was assigned to the lengthening of the Si-O bonds in the sub-stoichiometric oxide at the interface.

Therefore, irrespective of the film preparation, the silica films grown on Si crystals are vitreous in nature. The thinnest films (below $1 \mathrm{~nm}$ ) are ill-defined and apparently inhomogeneous. These findings can, in principle, be rationalized in terms of a large ( 25\% ) lattice mismatch between silica and $\mathrm{Si}$. In this respect, it was mentioned that the lattice parameters of $\mathrm{SiC}$ and $\mathrm{SiO}_{2}$ match within $\sim 5 \%$, thus allowing for an epitaxial $\mathrm{SiC} / \mathrm{SiO}_{2}$ interface. Note, that the formation of a high quality insulating oxide layer on $\mathrm{SiC}$ is a key requirement for metal-oxide-semiconductor devices based on this material. ${ }^{[23]}$

For the first time, the formation of a well-ordered silica layer on $\mathrm{SiC}$ has been reported by Heinz and co-workers ${ }^{[24]}$ by exposing the H-etched $\mathrm{SiC}(000-1)$ surface to air without any further treatment. The surface displayed a sharp $(\sqrt{3} \times \sqrt{3})$ R30 $0^{\circ}$ low energy electron diffraction (LEED) pattern. Even after leaving a sample in air for several hours the well ordered phase could be observed. The best fit (Pendry $R$-factor $=0.14$ ) was obtained for a model containing a $\mathrm{Si}_{2} \mathrm{O}_{3}$ monolayer on top of a bulk-like SiC substrate as shown in Figs. 4a,b. Further studies ${ }^{[25]}$ showed that the $(\sqrt{3} \times \sqrt{3}) \mathrm{R}^{\circ} 0^{\circ}$ structure on the Si-terminated, $\mathrm{SiC}(0001)$ surface is better described as a $\mathrm{Si}_{2} \mathrm{O}_{5}$ layer which connected via Si-O-Si linkages (R-factor of 0.20) as shown in Fig. 4c.

A DFT study ${ }^{[26]}$ showed that in the case of C-terminated SiC(000-1) surface, both the $\mathrm{Si}_{2} \mathrm{O}_{3}$ and the $\mathrm{Si}_{2} \mathrm{O}_{5}$ models have roughly the same formation energy, whereas the $\mathrm{Si}_{2} \mathrm{O}_{5}$ model is 
energetically much more favorable on the Si-terminated $\mathrm{SiC}(0001)$ surface. The calculated structural parameters were in excellent agreement with the LEED results ${ }^{[24-25]}$ and also angular resolved ultraviolet photoelectron spectroscopy (UPS) results. ${ }^{[27]}$

(a)



(b)

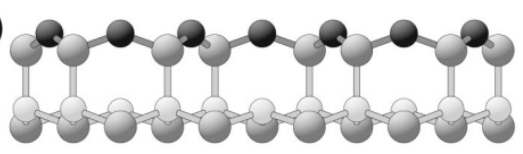

(c)

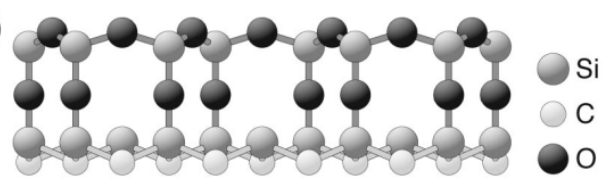

Figure 4. (a) Top view of the $\mathrm{Si}_{2} \mathrm{O}_{3}$ silicate structure on $\mathrm{SiC}(000-1)$. Dark shaded area indicates the $(1 \times 1)$, light shaded the $(\sqrt{3} \times \sqrt{3}) R 30^{\circ}$-unit cell. (b) Side-view projection of (a) along the (0110) direction (tilted forward). (c) Side view of the $\mathrm{Si}_{2} \mathrm{O}_{5}$ silicate adlayer structure on $\mathrm{SiC}(0001)$ in (01-10) projection. Reprinted with permission from ref. ${ }^{[25]}$ Copyright (1999) by the American Institute of Physics.

The Si-terminated SiC surface exhibits a significantly slower thermal oxidation rate than the C-terminated one, both grow in the amorphous state. The difference was linked to the presence of different amounts of the interfacial oxide $\mathrm{Si}_{4} \mathrm{C}_{4-\mathrm{x}} \mathrm{O}_{4}(\mathrm{x}<2)$ observed by angular resolved XPS. ${ }^{[28]}$ At the surface of $\mathrm{SiO}_{2}$, graphite and some $\mathrm{Si}_{4} \mathrm{C}_{4} \mathrm{O}_{4}$ were detected. IR studies of 
silica films grown on $\mathrm{SiC}$ as a function of film thickness ${ }^{[29]}$ showed frequency shifts below 10 nm for the TO mode, similar to that observed on Si-grown films (see Fig. 3b), but not for the LO mode.

\section{Silica films on metals}

The formation of adventitious silica on metal surfaces was reported in early days of "surface science" in the course of preparation of clean metal single crystal surfaces which commonly contained silicon as a bulk impurity. Its segregation and oxidation resulted in formation of oxidic structures which could sometimes be confused with or even erroneously referred to as the formation of a surface metal oxide, like in the case of "platinum oxide" (see e.g. refs. ${ }^{[30]}$ ) For example, at technically relevant pressures the formation of a "PtSiO" phase was observed on pure polycrystalline Pt even at room temperature. ${ }^{[31]}$ Adsorption studies of a Pt foil systematically contaminated with $\mathrm{Si}$ revealed that the resulting silica layers solely block adsorption of $\mathrm{CO}$ and $\mathrm{H}_{2}$ on Pt and do not produce any new adsorption sites. ${ }^{[32]}$

\subsection{Silica films on Molybdenum}

To the best of our knowledge, metal supported thin silica films were prepared for the first time by $\mathrm{Xu}$ and Goodman ${ }^{[33]}$ using $\mathrm{Mo}(110)$ as a substrate. Basically, a Mo(110) single crystal was chosen because of its relative ease of cleaning and the ability to thermally desorb the oxide thin films from the surface. Silica overlayers were grown at a relatively low temperature $(<$ $100^{\circ} \mathrm{C}$ ) by evaporating silicon in oxygen background, typically $\sim 1 \times 10^{-5}$ Torr. Based on AES data the $\mathrm{SiO}_{2}$ films could be as thin as $2 \AA{ }^{[34]}$ although it was unclear whether such ultrathin films were dense and uniform. Under these deposition conditions, the gas phase precursor to the 
$\mathrm{SiO}_{2}$ film was thought to be a gas-phase oxygen containing silicon species (likely $\mathrm{SiO}$ ), not gasphase silicon. The latter seems to dominate at lower oxygen pressures, in which further oxidation to $\mathrm{SiO}_{2}$ occurs on the $\mathrm{Mo}(110)$ surface. As deposited, the films were amorphous. Upon post-annealing, the films apparently become structurally more ordered, based solely on the narrowing of the respected infrared reflection-absorption (IRA) spectra dominated by features above $1100 \mathrm{~cm}^{-1}$ (Fig. 5). Since only the vibrations with a component normal to the surface are IR active for the films supported on metals, the IRA spectra exhibited only the features corresponding to the LO modes (Fig. 3a).

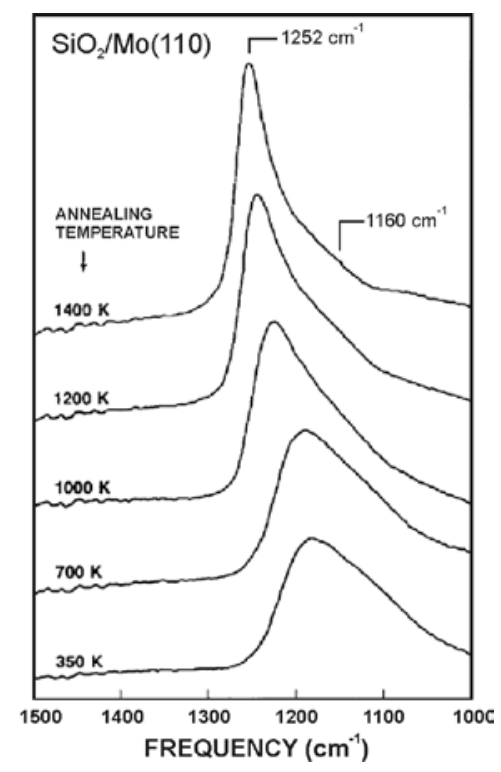

Figure 5. IRAS spectra for a $5 \mathrm{~nm}$-thick silicon dioxide film on $\mathrm{Mo}(110)$ annealed to the indicated temperatures. Reprinted from ref. ${ }^{[33 b]}$ Copyright (1993) with permission from Elsevier.

A few months later, Goodman's group reported the preparation of ultra-thin silica films on a Mo(100) surface. ${ }^{[35]}$ The films were characterized using XPS with respect to the chemical 
states of silicon and the composition of the film. It has been shown that the "as deposited" silicon oxide consisted predominantly of $\mathrm{SiO}_{2}$ with a small fraction of sub-oxides. Annealing to $\sim 1300$ $\mathrm{K}$ yielded a stoichiometric film of $\mathrm{SiO}_{2}$ which exhibited electronic and structural properties similar to those of vitreous silica.

However, the lattice parameters of both $\operatorname{Mo(110)}$ and $\operatorname{Mo(100)}$ surfaces do not fit those of the low-index silica surfaces of common silica polymorphs (depicted in Fig. 1b). This may explain the lack of long-range ordering in the resulting films. From all the stable single crystal surfaces of Mo, which has a body centered cubic structure, only Mo(112) could accommodate hexagonal surfaces without severe distortions. This choice has been proven successful, indeed. ${ }^{[36]}$ In the original recipe of Schroeder et al. ${ }^{[37]}$, the preparation consisted of four cycles of deposition of $\sim 0.5 \mathrm{ML}$ Si onto clean Mo(112) at room temperature and oxidation at $800 \mathrm{~K}$ in $5 \times 10^{-5}$ mbar $\mathrm{O}_{2}$ for $6 \mathrm{~min}$. The films were stepwise oxidized at higher temperatures, with the final oxidation being performed in $5 \times 10^{-6} \mathrm{mbar}_{2}$ at $1150 \mathrm{~K}$ for $20 \mathrm{~min}$. This preparation resulted in a closed film (as judged by $\mathrm{CO}$ and $\mathrm{H}_{2}$ adsorption experiments) which exhibited a sharp c(2×2)-Mo(112) LEED pattern (Fig. 6a), thus indicating the high degree of crystallinity. 

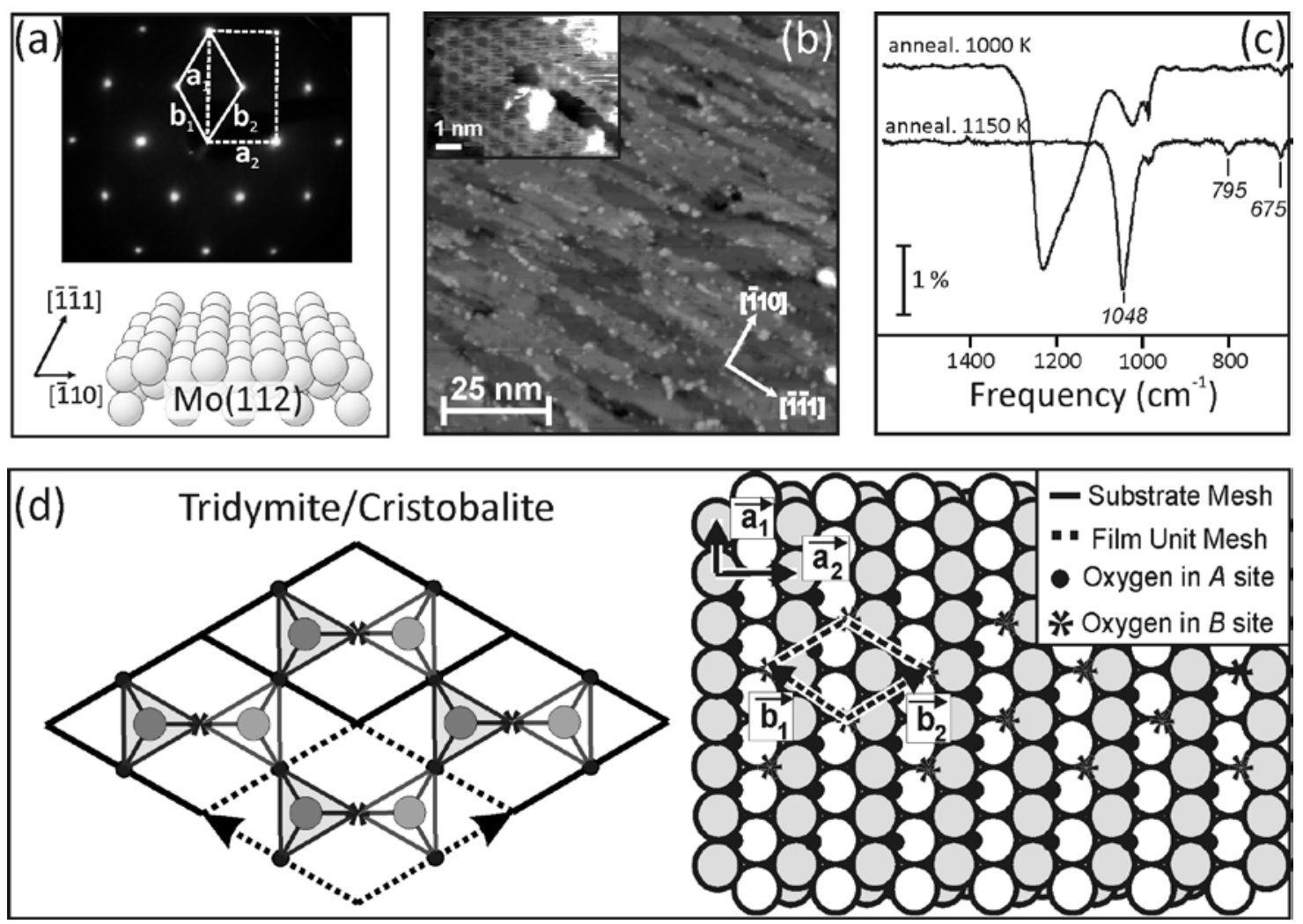

Figure 6. (a) Typical LEED pattern of a well-ordered silica film on Mo(112). A perspective view of the Mo(112) surface is shown, for clarity. Unit cells are indicated. (b) Large-scale STM image of the film annealed to $1150 \mathrm{~K}$. Inset shows high resolution image with a honeycomb-like structure. (c) IRAS spectra of silica films upon stepwise annealing to the indicated temperatures. (d) Model for a silica film on $\mathrm{Mo(112)}$ as suggested in ref. ${ }^{[37 \mathrm{~d}]}$

Figure 6a shows a perspective view of the Mo(112) surface which consists of closepacked atomic rows orientated along the [-1-11] direction which are separated by furrows in the [-110] direction, thus resulting in a rectangular surface unit cell with a spacing of $\mathrm{a}_{1}=2.73 \AA$ and $\mathrm{a}_{2}=4.45 \AA$, as indicated. The $\mathrm{c}(2 \times 2)$ structure of the silica overlayer on $\mathrm{Mo}(112)$ is very close to 
a hexagonal structure since the film unit cell vectors, $b_{1}$ and $b_{2}$, share an angle of $63^{\circ}$. The film unit cell length derived by LEED is $5.22 \AA$. A further spot-profile analysis LEED study ${ }^{[37 c]}$ revealed anti-phase domain boundaries and steps as the origin of the surface imperfections. Those defects attenuate considerably upon vacuum annealing at higher temperatures ( 1250 $\mathrm{K}),{ }^{[37 \mathrm{~d}]}$ which was used in the further preparations.

An XPS study ${ }^{[37 c]}$ of the annealed films showed silicon solely in the $\mathrm{Si}^{4+}$ state, whereas at least two oxygen species are present in the films, which were assigned to Si-O and interfacial SiO-Mo species. The XPS results also confirmed the absence of Si and Mo sub-oxides as well as Mo-silicides. UPS data ${ }^{[37 \mathrm{~d}]}$ were very similar to those of silica compounds with corner-sharing $\left[\mathrm{SiO}_{4}\right]$ tetrahedra as a building block.

Finally, an IRAS study ${ }^{[37 \mathrm{~d}]}$ revealed that upon high temperature annealing a strong band centered at $1048 \mathrm{~cm}^{-1}$ develops with simultaneous disappearance of bulk-like bands at around $1250 \mathrm{~cm}^{-1}$. This is observed for "as deposited" films or films annealed at lower temperatures (see Fig. 6c) and was also observed previously for silica films on $\operatorname{Mo}(100)^{[33 a]}$ (see Fig. 5). It is important to note that the high temperature treatments remove silanol (Si-OH) and other $\mathrm{OH}-$ containing surface species.

In meantime, Goodman’s group used metastable impact electron spectroscopy (MIES) in combination with UPS. ${ }^{[38]}$ The electronic structure of a $\mathrm{SiO}_{2}$ thin film on $\mathrm{Mo}(112)$ was found to be essentially identical to that of a $\mathrm{SiO}_{2}$ bulk crystal. The band gap of the $\mathrm{SiO}_{2}$ thin films was determined by MIES to be $8.8 \mathrm{eV}$, in agreement with the band gap of bulk $\mathrm{SiO}_{2}$.

Combined together the experimental results provided a solid basis for building a structure model. Several bulk-derived structures of different silica polymorphs were considered. In the case of HP-tridymite/ $\beta$-cristobalite (see Fig. 1b), both structures have a unit cell length of 5.05 
$\AA$, but a different stacking sequence (AB and $\mathrm{ABC}$ along [0001] and [111] directions of HPtridymite and $\beta$-cristobalite, respectively). The (0001) planes of the quartz have a unit cell length of $4.91 \AA$. All these structures have a considerable lattice mismatch with respect to the measured lattice constant of the pseudo-hexagonal silica overlayer on $\operatorname{Mo}(112)(=5.22 \AA$ ) (also seen in scanning tunneling microscopy (STM) images, see inset in Fig. 6b), and should therefore be distorted as compared with the bulk.

At this point it became clear that any model verification needs additional structural information and theoretical modeling. On the other hand, DFT calculations need input data about surface symmetry, its stoichiometry and thickness of an oxide overlayer, but the latter was the least known in that time. Indeed, determination of the film thickness with electron spectroscopies such as XPS and AES is based on the precise knowledge of a mean free path of electron in a material under study and therefore suffers from large uncertainty when applied to ultra-thin films where each layer counts. The reported values of Schroeder et al. ${ }^{[37 c]}$ and Goodman's group ${ }^{[38 a, 38 b]}$ scattered between 4 and $8 \AA$. Later, Chen et al. ${ }^{[39]}$ reported the value of $3 \AA$.

In their first DFT study of a silica film on Mo(112) Ricci and Pacchioni ${ }^{[40]}$ assumed a film thickness of about $1 \mathrm{~nm}$, which corresponds to three layers of corner-sharing [ $\left.\mathrm{SiO}_{4}\right]$ tetrahedra (Fig. 7). Since the silica film adheres with a layer of $\mathrm{O}$ atoms to the Mo substrate, the $\mathrm{SiO}_{2}$ polymorphs were cut so as to bind to the substrate with $\mathrm{O}$ atoms and to match the $\mathrm{c}(2 \times 2)$ pseudohexagonal structure of Mo(112). This resulted in (a) cleaving the bulk structure along the (111) direction of $\beta$-cristobalite and the (100) one for the other polymorphs and (b) expanding the silica lattice introducing a tensile strain in the film to match the substrate lattice dimensions. To saturate the surface bonds, cristobalite and tridymite were first terminated with isolated silanols, and quartz was terminated by germinal silanols. These calculations showed that $\beta$ - 
cristobalite is the most stable structure and exhibits the strongest adhesion energy to the Mo substrate. Then the dehydroxylated surface (to fit the experimental results showing the absence of $\mathrm{OH}$ at surface) undergoes a strong reconstruction which leads to the formation of twomembered silica rings that yield a vibrational band around $800 \mathrm{~cm}^{-1}$, i.e. close to $795 \mathrm{~cm}^{-1}$ found in the IRAS experiments (Fig. 6c).

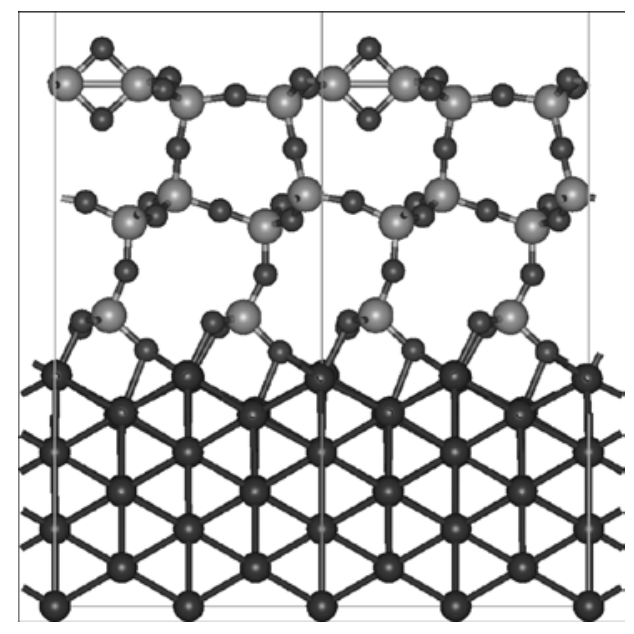

Figure 7. Structural model of the 3-layers thick $\mathrm{SiO}_{2}$ (cristobalite) film on $\mathrm{Mo}(112)$ exposing twomembered silica rings at the surface. (O: small black; Si: gray, Mo: large black). Reprinted with permission from ref. ${ }^{[40]}$ Copyright (2004) by the American Physical Society.

Almost simultaneously with this DFT study, Goodman and co-workers published a paper $^{[39]}$ addressing the atomic structure of the silica film on $\operatorname{Mo}(112)$, where, seemingly for the first time, the term "a monolayer silica film” was introduced. In addition to the original recipe of Schroeder et al. ${ }^{[37 a]}$, this group used another preparation, where the clean Mo(112) surface was first exposed to $5 \times 10^{-8}$ Torr $\mathrm{O}_{2}$ at $850 \mathrm{~K}$ to form the well-ordered $\mathrm{Mo}(112)-\mathrm{p}(2 \times 3) \mathrm{O}$ structure. Then less than $1 \mathrm{ML}$ Si was deposited, followed by annealing at $800 \mathrm{~K}$ in a $\sim 10^{-7}$ Torr $\mathrm{O}_{2}$ for 5 
min and then increasing the temperature to $1200 \mathrm{~K}$ for an additional 5 min. This deposition and annealing was repeated several times until a constant Si/Mo AES ratio was achieved, which was assigned to the film thickness approximately of $1 \mathrm{ML}$.

For a thicker film, the phonon bands at 496, 800 , and $1176 \mathrm{~cm}^{-1}$ were essentially the same as those measured for a quartz (0001) surface. ${ }^{[10]}$ Accordingly, ${ }^{[9 a]}$ these features were assigned to bending, symmetric stretching, and asymmetric stretching modes of Si-O-Si. At submonolayer and monolayer coverages, the phonon peaks at 496 and $1176 \mathrm{~cm}^{-1}$ are replaced by features at 320 and $1048 \mathrm{~cm}^{-1}$. At intermediate coverages, both spectral features exist, thus indicating two distinct Si-O species on the surfaces (see Fig. 8a). The shift from 1176 to $1048 \mathrm{~cm}^{-1}$ (i.e. 128 $\mathrm{cm}^{-1}$ ) is far too large to be attributed exclusively to effects of substrate coupling or to thinness of the film, i.e. typically in the order of $30-40 \mathrm{~cm}^{-1}$ (see Fig. 3). Instead, based on the HREELS and IRAS data available in the literature for $\mathrm{Si}-\mathrm{O}-\mathrm{Si}(\mathrm{Al}, \mathrm{Ti}, \mathrm{Zr})$ systems, the $1048 \mathrm{~cm}^{-1}$ peak was assigned to a Si-O-Mo asymmetric stretching mode. ${ }^{[39]}$
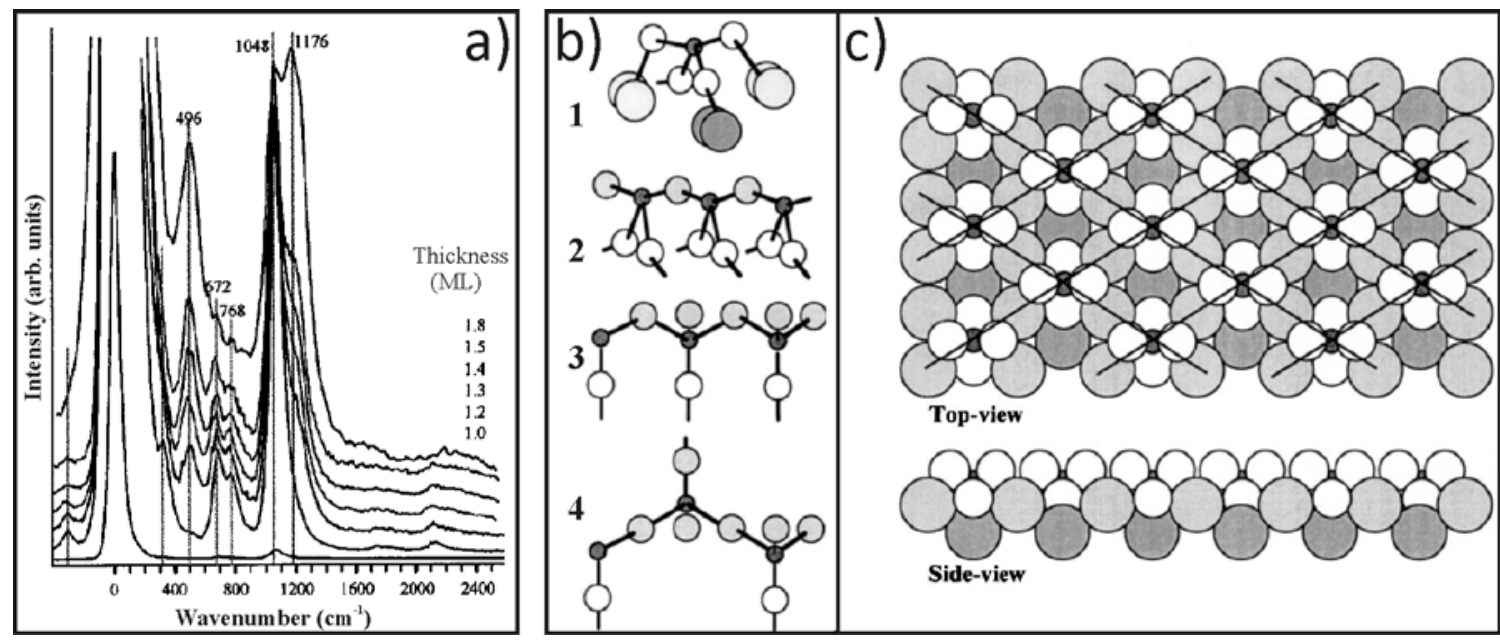
Figure 8. (a) HREELS spectra of the thin silica films on Mo(112). (b) Schematic of $\left[\mathrm{SiO}_{4}\right]$ binding geometries with the substrate Mo atoms: (1) isolated [ $\left.\mathrm{SiO}_{4}\right]$ clusters; (2) chain-like structure; (3) two-dimensional network; (4) two-dimensional network for HP-tridymite and $\square$ cristobalite models. (c) Top and side view of the for c(2x2)-[SiO 4$]-\mathrm{Mo}(112)$ "cluster" model. Reprinted with permission from ref. ${ }^{[39]}$ Copyright (2004) by the American Physical Society.

In their structure modeling Chen et al. considered different binding geometries of $\left[\mathrm{SiO}_{4}\right]$ to the Mo surface atoms as schematically shown in Fig. 8b. In order to explain the absence of the $1076 \mathrm{~cm}^{-1}$ band assigned to the asymmetric Si-O-Si stretching mode in silica, which (under constraints of the metal selection rules) suggests that only Si-O-Si species with no component normal to the surface exist, Chen et al. were left with the model of isolated $\left[\mathrm{SiO}_{4}\right]$ clusters arranged in $\mathrm{c}(2 \times 2)$ manner on $\mathrm{Mo}(112)$ as shown in Fig. 8c (hereafter referred to as a "cluster model"). The authors also noted that there may be additional $\mathrm{O}$ atoms located among the silica cluster and bonded only to the Mo atoms which may account for phonon band at $672 \mathrm{~cm}^{-1}$.

However, in a follow-up study ${ }^{[41]}$ Goodman's group, in fact, disowned the cluster model. The authors presented O1s XPS data collected for a monolayer $\mathrm{SiO}_{2}$ film. There were two different oxygen species with binding energies of approximately 530.3 and $532.0 \mathrm{eV}$, which were assigned to $\mathrm{Si}-\mathrm{O}-\mathrm{Mo}$ and $\mathrm{Si}-\mathrm{O}-\mathrm{Si}$ species, respectively, in the chain-like structure (model 2 in Fig. 8b), in which two of the $\mathrm{SiO}_{2}$ oxygen atoms are tetrahedrally bonded to the Mo substrate, with the remaining two oxygen atoms bridging the neighboring silicon atoms to form a chain.

Apparently, the uncertainty in the identification of the atomic structure of crystalline silica films could result from the different preparations used. Santra et al. ${ }^{[42]}$ addressed this issue 
using LEED and STM in attempt to optimize the parameters crucial to the preparation of highquality $\mathrm{SiO}_{2}$ films on $\mathrm{Mo}(112)$, i.e., silicon deposition flux, oxidation time and exposures, and the temperature/time of the final anneal. Comparative STM and LEED studies showed that a sharp $\mathrm{c}(2 \times 2)$ LEED pattern is insufficient evidence for a high-quality, flat film. The large-scale STM images of the films obtained in this work showed a stripe-like morphology oriented along the Mo(-1-11) direction, which was very similar to that shown in Fig. 6 b by Schroeder et al. ${ }^{[37 d]}$ On the other hand, STM images published later by the same group ${ }^{[41,43]}$ revealed a much smoother surface, although the films were prepared identically.

Electronic and vibrational properties of $\mathrm{SiO}_{2} / \mathrm{Mo}(112)$ was addressed by Wendt et al. ${ }^{[44]}$ using polarization modulation (PM) IRAS and UPS/MIES. The authors focused on the film synthesis using various preparation methods. In addition to the method of deposition-oxidation cycles on $\mathrm{O}(2 \times 3)-\mathrm{Mo}(112)$ previously introduced by Chen et al. ${ }^{[39]}$, the second method included a single step deposition and annealing in oxygen at high temperatures. Regardless of the preparation recipe, the electronic and vibrational properties of monolayer films were best rationalized by a surface dominated by Si-O-Mo linkages. For such films a single vibrational feature at $1048-1057 \mathrm{~cm}^{-1}$ was detected with PM IRAS and assigned to the Si-O-Mo linkages. For coverages greater than one monolayer, the formation of Si-O-Si linkages in the second layer of the $\mathrm{SiO}_{2}$ films was observed. In the well-ordered monolayer film, UPS and MIES spectra revealed the $\mathrm{O}(2 \mathrm{p})$ nonbonding band splitting in two well-resolved features at 7.6 and $\sim 6.3 \mathrm{eV}$, consistent with two distinct $\mathrm{O}(2 \mathrm{p})$ nonbonding orbitals at the surface. The fine structure of the nonbonding band was, again, attributed to isolated $\left[\mathrm{SiO}_{4}\right]$ tetrahedra attached via $\mathrm{O}$ atoms to the substrate. 
At this point it became more and more evident that only a multi-technique approach, including high-resolution STM, and electronic and vibrational spectroscopies applied to the same sample, in combination with the state-of-the art DFT calculations can elucidate the atomic structure of the monolayer silica film on Mo(112). This step forward was made in 2005, when two groups ${ }^{[45]}$ almost independently came to the conclusion that the structure of a silica film on $\mathrm{Mo}(112)$ consisted of a two-dimensional network of corner-sharing $\left[\mathrm{SiO}_{4}\right]$ tetrahedra. It is fair to say that this “2D-network” model was considered, but rejected, by Goodman and co-workers. ${ }^{[39]}$

The experiments were performed in an UHV chamber equipped with LEED, UPS/XPS, IRAS and STM. Following the recipe of Chen et al. ${ }^{[39]}$ the films were prepared by exposing the clean $\mathrm{Mo}(112)$ surface to $5 \times 10^{-8}$ mbar $\mathrm{O}_{2}$ at $900 \mathrm{~K}$, thus resulting in $\mathrm{p}(2 \times 3) \mathrm{O}-\mathrm{Mo}(112)$ surface. (NB: The proposed atomic structure of this surface was later revisited by $u^{[46]}$ ). Subsequently, approximately $1 \mathrm{ML}$ of $\mathrm{Si}$ was deposited in one step at the same oxygen pressure and temperature. The film was then annealed in UHV at $\sim 1250 \mathrm{~K}$ for 5 min. The annealing temperature has turned out to be critical. Based on the STM studies, overheating leads to a partial sublimation of the film, while annealing at the lower temperatures results in the presence of illdefined silica particles on top of the ordered film.

Figures 9 shows large-scale STM images and respective IRAS spectra of the "as deposited" and annealed films. It is clear that a well-ordered films is characterized by an extremely sharp $\left(\mathrm{FWHM}=12 \mathrm{~cm}^{-1}\right.$ ) and strong band at around $1060 \mathrm{~cm}^{-1}$ and weak, but clearly visible bands at 770 and $675 \mathrm{~cm}^{-1}$. All peaks are red-shifted by 41,7 and $19 \mathrm{~cm}^{-1}$, respectively, when measured on the films prepared with ${ }^{36} \mathrm{O}_{2}$ isotope. ${ }^{[45 a]}$ Silica deposition on top of the annealed films immediately results in the broad band at $\sim 1240 \mathrm{~cm}^{-1}$ also seen for the "as deposited" samples, and as such is attributed to silica particles and their aggregates, ${ }^{[4]}$ in 
agreement with the previous results. ${ }^{[33 b, 39,44]}$ Subsequent annealing to $1250 \mathrm{~K}$ restores both the morphology and IRAS spectrum of the atomically flat film, thus indicating "self-limited” growth of the monolayer film via sublimation of the silica clusters at high temperatures. The crystalline films show wide terraces (Fig. 9b), with steps of $1.2 \AA$ in height corresponding to monoatomic steps of the Mo(112) substrate underneath the film.
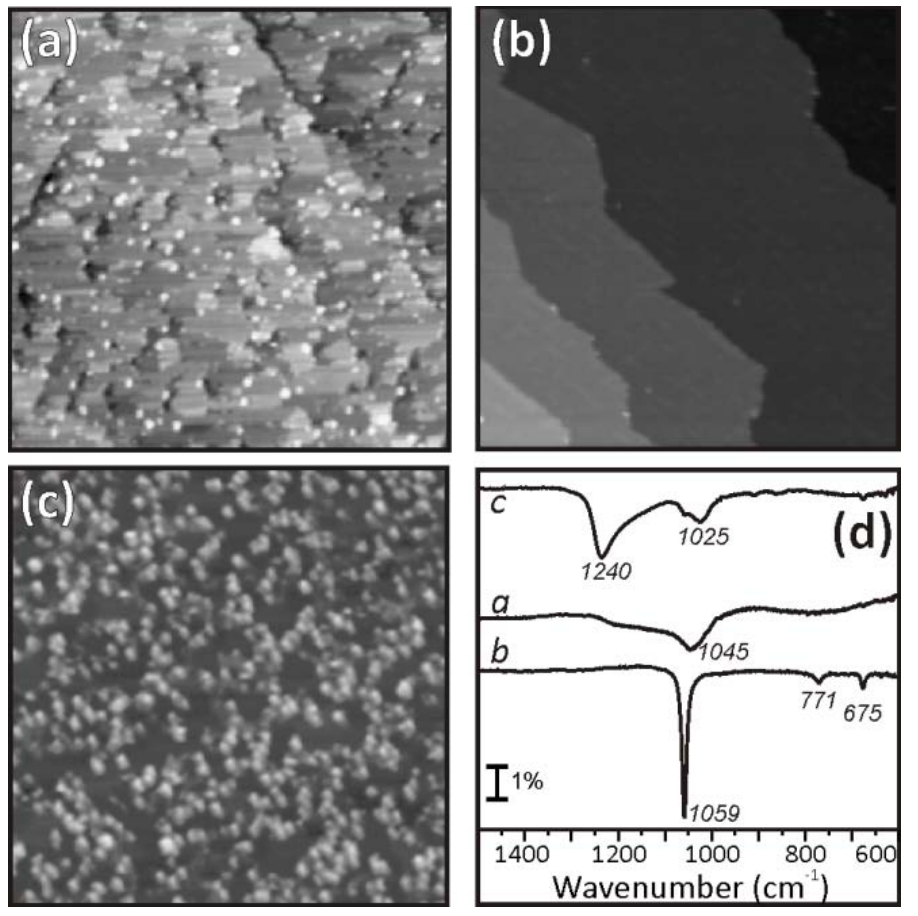

Figure 9. STM images (a-c) and IRAS spectra (d) of the silica film: (a) as deposited at $900 \mathrm{~K}$; (b) subsequently annealed in UHV at $1250 \mathrm{~K}$; (c) with additionally deposited silica on top of the film (b). Image size is $100 \mathrm{~nm} \times 100 \mathrm{~nm}$.

An XPS study showed only a single peak in the Si 2p region with a binding energy (BE) of $103.2 \mathrm{eV}$, which is characteristic for the $\mathrm{Si}^{4+}$ oxidation state. In contrast, the $\mathrm{O}$ 1s region revealed two different chemical environments for oxygen, with $\mathrm{BE}$ at $532.5 \mathrm{eV}$ and $531.3 \mathrm{eV}$. 
These two states are much better resolved using synchrotron light (Fig. 10). ${ }^{[48]}$ The BE values are considerably higher than the $530.5 \mathrm{eV}$ observed for the $\mathrm{MoO}_{\mathrm{x}}$ oxide layers on $\mathrm{Mo}(112)$ and therefore cannot be assigned to Mo-oxide formation underneath the film. The intensity ratio of two $\mathrm{O}$ species at higher and lower BEs in well-ordered films is found to be about 3:2.
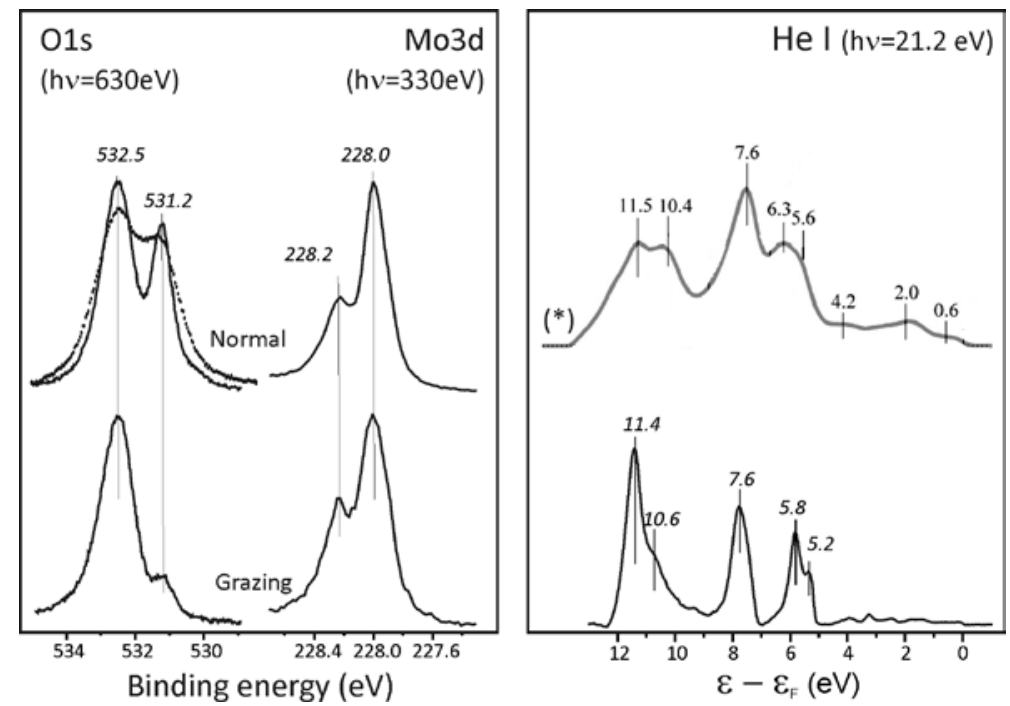

Figure 10. (Left panel) XPS spectra (O1s and Mo3d core levels) of a silica film on Mo(112) obtained with the synchrotron light at photon energies as indicated. The dotted line shows the O1s spectrum obtained with a conventional x-ray source, for comparison. (Right panel) UPS spectrum (He I) of a crystalline silica film on Mo(112). Spectrum $\left(^{*}\right)$ from ref..$^{[44]}$ is shown for comparison.

The presence of very narrow bands in an UPS spectrum (Fig. 10) confirms the high crystallinity of the films grown. The signals between 4 and $9 \mathrm{eV}$ are commonly attributed to nonbonding O2p derived valence levels, and the signals between 10 and $12 \mathrm{eV}$, in the region of Si-O bonding states observed for silica films on $\mathrm{Si}$, are attributed to the hybridized O2p-Si3s,3p 
bonding states. Wendt et al. ${ }^{[44]}$ previously assigned a band at $\sim 11.4 \mathrm{eV}$ to the Si-O-Si bonds. Consequently, the band at $10.6 \mathrm{eV}$ was attributed to the Si-O-Mo bond, where the interface O atoms are thought to accumulate electron density from the Mo substrate. Some differences between UPS spectra from different publications could be traced to the differences in film preparations and film quality, which is hard to compare without STM. Indeed, STM studies ${ }^{\text {[49] }}$ revealed that atomically flat films can be produced only after annealing at temperatures above $1200 \mathrm{~K}$, which was not always the case in the previous works.

Perhaps the most crucial piece of structural information originated from the atomically resolved STM images. Figure 11a clearly shows a honeycomb-like structure with a $\sim 5.4 \AA$ periodicity, and line defects all running along the [-110] direction of $\mathrm{Mo}(112)$. These lines, imaged as elongated depressions larger than in the regular hexagons (i.e. 6-membered rings), can easily be formed by a half-lattice shift in the [-1-11] direction between two domains, thus resulting in alternating 8- and 4-membered rings of [ $\left.\mathrm{SiO}_{4}\right]$ tetrahedra, as depicted in Fig. 11c. Certainly, the image contrast may depend on the bias and tip conditions (compare Figs. 11c and inset in Fig. 11b) showing either $\mathrm{O}$ or Si atoms as protrusions. Nonetheless, these results favor the 2D model of the silica film which is formed by a monolayer network of corner-sharing $\left[\mathrm{SiO}_{4}\right]$ tetrahedra, where one corner of each tetrahedron is connected to the Mo(112) via Si-O-Mo linkages inferred by the IRAS and XPS results.
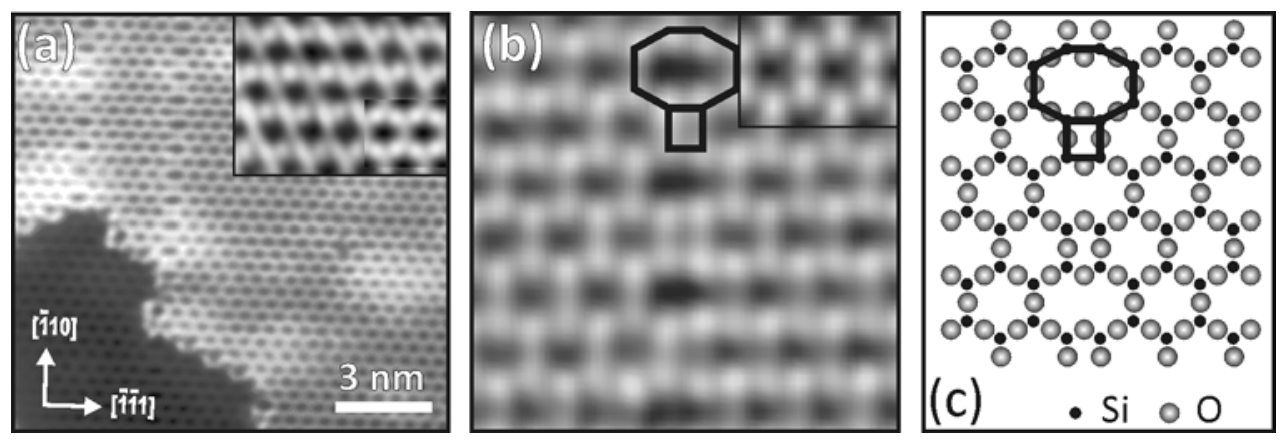
Figure 11. High resolution STM images of a crystalline silica film on Mo(112). Depending on the tunneling conditions the $\mathrm{O}$ and $\mathrm{Si}$ atoms are imaged as protrusions in (a) and (b), respectively. The line defect, running along the [-110] direction, in the image (a) is atomically resolved in the image (b). Panel (c) displays a top-view of the monolayer network of cornersharing $\left[\mathrm{SiO}_{4}\right]$ tetrahedra, which readily explains the STM contrast in the image (b) by introducing a half-lattice shift between two domains. Inset in (a) and the image (b) are superimposed with the simulated STM images based on the DFT-optimized structural model shown in Fig. 12.

In order to examine the relative stability of the possible models and their agreement with the experimental results, DFT calculations were performed by Sauer and co-workers ${ }^{[45 a]}$ for numerous models consisting of single and double layers of $\left[\mathrm{SiO}_{4}\right]$ tetrahedra, which do not contain dangling $\mathrm{Si}-\mathrm{O}$ bonds or edge-shared $\mathrm{SiO}_{4}$-tetrahedra (the computational details are described in ref. ${ }^{[50]}$ ). The most stable structure was found to be the monolayer structure with a composition of $\mathrm{SiO}_{2.5}$, which is formed via a network of corner-sharing $\left[\mathrm{SiO}_{4}\right]$ tetrahedra (Fig. 12a), with one corner of each tetrahedron forming a Si-O-Mo bond. Furthermore, the DFT study could address the film registry with respect to the Mo(112) surface, since the registry strongly affected the computed IRA frequencies (by $~ 75 \mathrm{~cm}^{-1}$ ). Three IRAS active modes were obtained: The asymmetric Si-O-Mo stretching at $1061 \mathrm{~cm}^{-1}$ originating from the Si-O bond pointing downwards to the Mo substrate; the second mode at $778 \mathrm{~cm}^{-1}$ from the Si-O-Si symmetric stretching coupled with Si-O-Si bending and the third mode at $670 \mathrm{~cm}^{-1}$ from a coupling of Si-OSi bending modes. The calculations also nicely reproduced the experimentally observed isotopic 
shifts for the films grown with ${ }^{36} \mathrm{O}_{2}$. Furthermore, this model explained the BE shift $(1.3 \mathrm{eV})$ and intensity ratio (3:2) for the $\mathrm{O} 1$ s core level of the oxygen ions in the top-most layer, $\mathrm{O}(\mathrm{Si})$, and the interfacial $\mathrm{O}(\mathrm{Mo})$ atoms, having bonds both to Si and Mo. Finally, STM image simulations nicely agree with experimental high-resolution STM images (see insets in Fig. 11).
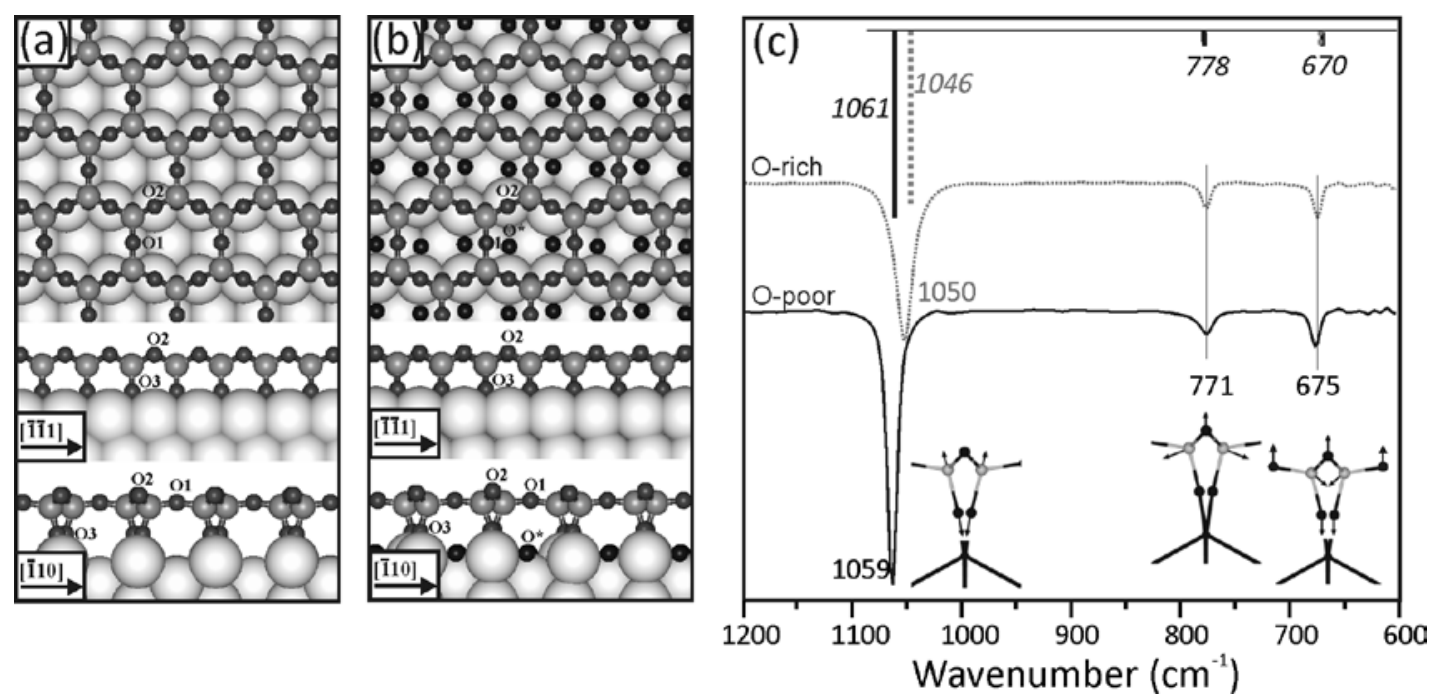

Figure 12. Top and cross views of “O-poor" (a) and "O-rich” (b) $\mathrm{SiO}_{2.5} / \mathrm{Mo}(112)$ films. (O: small dark; Si: large gray). (c) Vibrational modes and calculated frequencies for the models (a) and (b) shown as bars with their height proportional to the intensity. The experimental spectra of the silica films annealed in UHV (a so called “O-poor” film) and in oxygen (“O-rich”) are shown, for comparison.

The network model described so far contains no oxygen atoms other than those involved in the O-Si-O and Si-O-Mo linkages. In principle, the open honeycomb-like structure of the film allows $\mathrm{O}$ atoms to reside "inside" the rings in different adsorption sites on the Mo(112) surface. These so-called "O-rich" structures were examined by DFT. ${ }^{[51]}$ The calculations revealed that the 
stability of the "O-rich" phases considerably increases at high chemical potential of oxygen (e.g., high pressure, low temperature). A careful analysis of the experimental IRAS and XPS spectra in the monolayer films prepared by high temperature annealing either in UHV or $10^{-6}$ mbar $\mathrm{O}_{2}$ showed, indeed, small but detectable changes, which agreed well with the theoretical predictions (Fig. 12c). Due to subtle differences between properties of the O-poor and O-rich films, the existence of the latter might not have been discovered without the aid of theory. The observation of a variety of different structures may explain the somewhat controversial results reported from different groups and experimental setups as discussed above. It became evident that the precise atomic structure may strongly depend on film preparation.

As already mentioned, Giordano et al. ${ }^{[45 b]}$ basically came to the same conclusions about the atomic structure of the crystalline silica films. Again, their DFT study showed that neither the cluster nor the chain-like model could account for the measured vibrational spectra. Later, Yakovkin ${ }^{[52]}$ reported calculations for the $\left[\mathrm{SiO}_{4}\right]$ cluster model which resulted in a vibrational mode at $1044 \mathrm{~cm}^{-1}$, which was then used by Chen et al. ${ }^{[53]}$ to theoretically support the cluster model. However, these calculations were based on a small molecular model of the surface containing just one $\left[\mathrm{SiO}_{4}\right]$ unit, which could not, adequately, describe the Mo conduction band electrons and the coupling between vibrational modes of the silica layer and the substrate. Indeed, the DFT calculations of the cluster model by Todorova et al. ${ }^{[49]}$ and Giordano et al. ${ }^{[45 b]}$ did not reveal any IRAS active bands at frequencies higher than 706 and $840 \mathrm{~cm}^{-1}$, respectively.

It was tempting to directly measure the thickness of the silica films by STM, e.g. by preparing films which partially covered the surface. Surprisingly, the STM images revealed a strip-like surface morphology ${ }^{[54]}$ which somewhat resembled those reported in early publications [37d, 42] of the films thought to be dense. When coalesced, the stripes form islands with a 
honeycomb-like structure that is virtually identical to that observed on the fully covered monolayer films (Fig. 13a). The STM protrusions on the stripes and on the island surfaces were found to be in perfect registry, which implies the same nature of protrusions. Interstingly, the spacing between stripes $(13.4 \AA)$ is the same as on the $\mathrm{p}(2 \times 3) \mathrm{O}-\mathrm{Mo}(112)$ reconstructed surface used as a substrate for Si deposition. This finding suggests that the structure of silica stripes is driven by a reconstructed metal surface formed prior to the Si deposition.
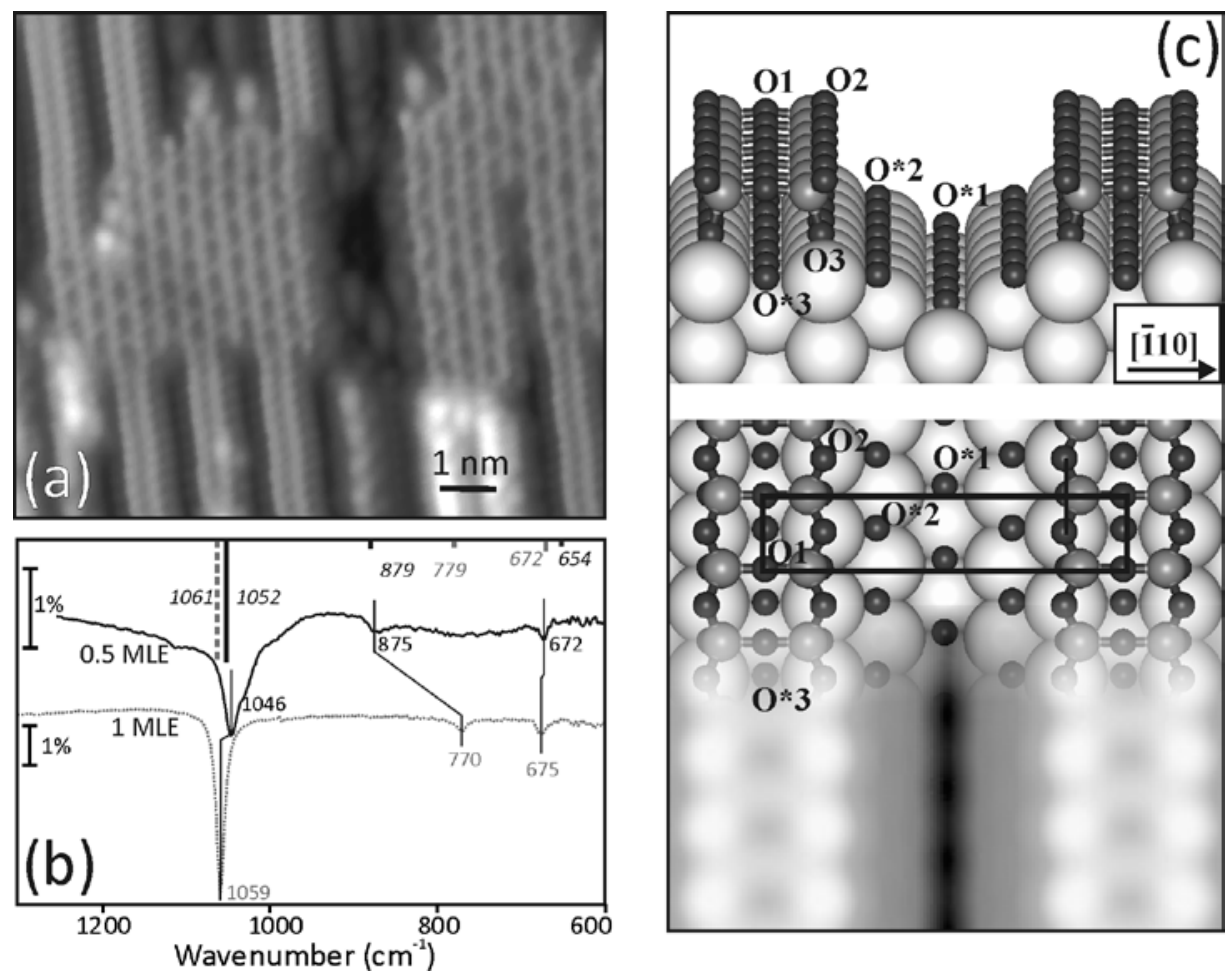

Figure 13. (a) High-resolution STM image of a silica film on Mo(112) at sub-monolayer coverages. (b) IRAS spectra for the fully (1 MLE) and partially (0.5 MLE) covered silica films. (c) Perspective and top views of the thermodynamically most stable structure of silica stripes on Mo(112). A simulated STM image is superimposed with the structure. The $(1 \times 3)$ surface unit 
cell is indicated. The calculated frequencies for this structure are shown in (b) as bars in the same color code as the experimental spectra.

The proposed model is shown in Fig. 13c. This structure explains the STM image contrast. Even stronger evidence for the proposed structure came from the analysis of lattice vibrations, which were previously shown to be sensitive to the silica structure and, in particular, to the registry between silica and the Mo substrate. ${ }^{[49]}$ Figure $13 \mathrm{~b}$ shows that the DFT computed band positions and intensities are in excellent agreement with the experimental IRAS spectra. For the silica stripes, the spectra revealed a small red-shift of the principal phonon at $1059 \mathrm{~cm}^{-1}$ to $1046 \mathrm{~cm}^{-1}$, whereas the mode at $770 \mathrm{~cm}^{-1}$ in the films is considerably $\left(\sim 100 \mathrm{~cm}^{-1}\right)$ blue-shifted to $875 \mathrm{~cm}^{-1}$. This large shift is due to the fact that this mode, i.e. coupling the $\mathrm{Si}-\mathrm{O}-\mathrm{Si}$ symmetric stretching and $\mathrm{Si}-\mathrm{O}-\mathrm{Si}$ bending mode in the 2D-film, has less $\mathrm{Si}-\mathrm{O}-\mathrm{Si}$ bending character in the 1D-stripe. Again, the results provided further indirect evidence for the 2Dnetwork model of the monolayer silica film.

Nonetheless, Chen and Goodman ${ }^{[53]}$ continued the discussions on the structure of the monolayer silica films on $\mathrm{Mo}(112){ }^{[55]}$ The key point was the nature of vibrational modes measured for the silica films by IRAS and HREELS. Basically, the absence of the band at $\sim 1176 \mathrm{~cm}^{-1}$ (assigned to asymmetric Si-O-Si stretching) in the monolayer films has been used as a central argument to conclude that the monolayer film does not exhibit Si-O-Si bonds at all and hence consists of exclusively $\left[\mathrm{SiO}_{4}\right]$ clusters with the $\mathrm{Si}-\mathrm{O}-\mathrm{Mo}$ linkages only.

These issues were explicitly addressed by Kaya et al. ${ }^{[48]}$. A very detailed analysis of the vibrational spectra based on symmetry considerations was carried out to explain how these 
spectra relate to those observed for the "bulk"-like systems, e.g. thick silica films and single crystals.

Recently, further support for the network model of the monolayer silica film came from studies by Seifert et al., ${ }^{[56]}$ using fast hydrogen and helium atom scattering at a grazing angle of incidence. The experimental results exclude the cluster model and nicely fit the network structure. Finally, a very recent I/V LEED study ${ }^{[57]}$ showed again excellent agreement (R factor 0.14) with the network model.

Having established the atomic structure of a crystalline silica film on Mo(112), one may raise the question: how is this surface related to surfaces of silica polymorphs? In this context, it is noteworthy that a similar surface structure was predicted by molecular-dynamics simulations for the reconstructed $\alpha$-quartz(0001) surface as the most stable of all investigated structures, and as such was proposed as a model of the dry reconstructed (0001) surface obtained upon cleaving. ${ }^{[58]}$ Such a surface was modelled via densification of the two uppermost layers of $\left[\mathrm{SiO}_{4}\right]$ tetrahedral units, with 6-membered (in top view) and 3-membered (cross view) rings that do not exist in bulk $\alpha$-quartz. The free-standing silica double layer is computed to have a lattice constant of $5.24 \AA^{[59]}$ or $5.30 \AA \AA^{[60]}$ which shows a considerable mismatch with respect to $\alpha$ quartz(0001) (= $4.91 \AA)$, such that its formation on real quartz surfaces is most likely prevented. In contrast, a silicate monolayer was observed on $\mathrm{SiC}(000-1)$ and (0001) surfaces $^{[24]}$ (see Fig. 4) leading to the hexagonal $(\sqrt{3} \mathrm{x} \sqrt{3}) \mathrm{R} 30^{\circ}$ structure with a lattice constant of $5.25 \AA$, i.e. very close to that calculated for the unsupported silica layer. On the other hand, the silicate monolayer hardly fits the $\mathrm{Mo}(112)$ surface with a rectangular unit cell (5.46 $\AA$ × $8.92 \AA$ ), and is, therefore, accompanied by a lattice extension along the Mo[-1-11] direction to $5.46 \AA$ as compared to the free-standing layer. Despite this distortion, the monolayer silica films on Mo(112) are perfectly 
ordered, as shown by STM. The reason is the very strong Si-O-Mo bond that stabilizes this particular structure. Consequently, the metal support induces considerable changes in the electronic structure. A DFT analysis of valence band structures suggested that the silica film on Mo(112) must be considered as a novel oxidic surface and not as another incarnation of a "dense" surface on bulk $\alpha$-quartz. ${ }^{[59]}$

As expected, the O-terminated silica film on $\mathrm{Mo(112)}$ is essentially inert towards any gases. Moreover, even after exposure to air, the film surface is fully recovered by a thermal flash in UHV to $\sim 1000 \mathrm{~K}$. The adsorption properties of the monolayer silica film on Mo(112) were primarily studied with respect to water, since silanol (Si-OH) groups are very important in catalysis where silica is involved either as a support for catalyst particles or as a reactive surface. ${ }^{[2]}$ Wendt et al. ${ }^{[61]}$ studied the interaction of water at low temperatures using MIES, UPS, HREELS, TPD and work function measurements. The data suggested the growth of threedimensional water clusters even at low coverage from the onset, i.e. the water-water hydrogen bonding is stronger than the water-silica interaction. No evidence was found for dissociation of water.

No water dissociation was found either in our own studies of the defect-free silica films by TPD, IRAS and XPS with synchrotron radiation. ${ }^{[62]}$ In contrast to adsorption at $100 \mathrm{~K}$, where water followed zero-order desorption kinetics, when adsorbed at $140 \mathrm{~K}$, water exhibited pseudofirst-order kinetics and induced a strong blue shift of the principal silica phonon at $\sim 1060 \mathrm{~cm}^{-1}$. Even larger spectral shifts were observed for $\mathrm{D}_{2} \mathrm{O}$ adsorption. The results were rationalized in terms of the formation of a crystalline ice film at $140-150 \mathrm{~K}$ in contrast to an amorphous solid water film at $100 \mathrm{~K}$. This film is well-ordered and exhibits a long-range ordered $\mathrm{c}(2 \times 2)$ structure with respect to the silica surface or a $c(6 \times 2)$ structure when referred to $\operatorname{Mo}(112)$. The epitaxial 
growth of ice is probably favored by the small lattice mismatch. Indeed, the $\mathrm{O}-\mathrm{O}$ distance in the ice Ih phase is $2.75 \AA$ ( $2.61 \AA$ when projected onto the (0001) plane), which fits fairly well to the O-O distance in the topmost O-layer of the silica film (2.73 $\AA$ ). The proposed model consists of a water bilayer, commonly considered for adsorption on metal surfaces, but still needs verification by theoretical calculations. For comparison, DFT calculations of water adsorption on hydroxylated $\alpha$-quartz (0001) surfaces found an ordered hexagonal water layer with a flat bilayer structure, compared with the basal plane of ice Ih. The so-called "H-down" configuration was found to be energetically more favorable than the "H-up" configuration. ${ }^{[63]}$

Attempts to grow well-ordered multilayer silica films on Mo(112) resulted in silica overlayers with smooth but amorphous surfaces, i.e. showing no LEED pattern, although the films exhibited sharp phonon spectra. ${ }^{[64]}$ Annealing these "thick" films at higher temperatures led either to film decomposition or back to the monolayer film.

The history of establishing the atomic structure of the crystalline silica films on $\operatorname{Mo}(112)$, discussed here in great detail, illustrates that it is very important to make use of a combination of several experimental techniques applied to the same sample. This system also exemplifies the critical role of theoretical calculations in determining the atomic structure of metal supported oxide films where a substrate is critically involved in the stabilization of the oxide structure.

\subsection{Silica films on Nickel and Palladium}

Along with the preparation of silica films on Mo(112), there were a few other attempts to grow crystalline silica films on metal supports. Kundu and Murata ${ }^{[65]}$ reported the growth of a single-crystal $\mathrm{SiO}_{2}$ film on $\mathrm{Ni}(111)$. The surface structure of the films was studied by LEED, 
AES and STM. Three-dimensional structural information was obtained by grazing-incidence $\mathrm{x}$ ray diffraction (GIXD) analysis along a reciprocal lattice rod known as a Crystal Truncation Rod. The interfacial structure between $\mathrm{SiO}_{2}$ and $\mathrm{Ni}(111)$ phases was characterized by in-plane GIXD.

For the preparation of crystalline silica film, first a Si film of about $3 \mathrm{~nm}$ in thickness was deposited in UHV onto the clean $\mathrm{Ni}(111)$ surface at $300 \mathrm{~K}$. This was followed by oxidation at $2 \times 10^{-7}$ Torr $\mathrm{O}_{2}$ in the presence of atomic hydrogen for $1 \mathrm{~h}$ at $350^{\circ} \mathrm{C}$. Finally, the sample was annealed at $800^{\circ} \mathrm{C}$ in the oxygen ambient for $10 \mathrm{~min}$. The resulting silica film was about $4 \mathrm{~nm}$ in thickness as measured by optical ellipsometry, i.e. less than the value expected from the deposited Si thickness, which was attributed to partial evaporation of volatile $\mathrm{SiO}_{\mathrm{x}}$ species. The film showed a sharp LEED pattern assigned to a $(\sqrt{3} \times \sqrt{ } 3)$ R3 $30^{\circ}$ structure. Atomic hydrogen exposure during the initial growth of the amorphous silica film and an ambient oxygen atmosphere during annealing of this film play important roles for crystallinity of these films. Except the above-described recipe, all other attempts did not result in ordered films. Also, the preparation of thinner films was not possible due to the formation of a polycrystalline silicide phase at the $\mathrm{SiO}_{2} / \mathrm{Ni}(111)$ interface.

GIXD analysis of a crystalline film led the authors to conclude that this silica film is $\square$ quartz ( $\mathrm{a}=5.01 \AA$ ) which better fits the surface of $\mathrm{Ni}(111)\left(2 \times \mathbf{a}_{\mathrm{Ni}(111)}=4.984 \AA\right)$ than $\alpha$-quartz (4.913 $\AA$ ). Nonetheless, the surface structure of the films was not identified.

Recently, Zhang et al. ${ }^{[66]}$ reported the preparation of thin silica films on $\operatorname{Pd}(100)$. The films were characterized by XPS/UPS, HREELS, STM and ion scattering spectroscopy (ISS). The silica films were grown by depositing $\mathrm{Si}$ onto a $\operatorname{Pd}(100)$ substrate kept at $500 \mathrm{~K}$ in $1 \times 10^{-3} \mathrm{~Pa}$ of $\mathrm{O}_{2}$. The film thickness was measured by attenuation of the Pd 3d XPS signal and 
controlled by the Si deposition time. The calculated silica film thicknesses ranged between 0.4 and $6.5 \mathrm{~nm}$.

An XPS study showed no Pd-silicide formation at the $\mathrm{SiO}_{2} / \mathrm{Pd}(100)$ interface, in contrast to the $\mathrm{SiO}_{2} / \mathrm{Ni}(111)$ system (see above). For the 2.8 and $6.5 \mathrm{~nm}$-thick silica films, the $\mathrm{Si} 2 \mathrm{p}$ spectra presented a small shoulder peak at low binding energy, most likely originating from silicon suboxide. The UPS results indicated that these films have a similar electronic band structure compared to that of crystalline bulk $\mathrm{SiO}_{2}$, although the $\mathrm{O}$ 2p non-bonding states were not resolved in this study. HREELS spectra showed losses at 458, 827 (as a shoulder) and $1106 \mathrm{~cm}^{-1}$ (Fig. 14a), which were assigned to the bending, symmetric stretching, and asymmetric stretching modes of $\mathrm{Si}-\mathrm{O}-\mathrm{Si}$, respectively. The most prominent $1106 \mathrm{~cm}^{-1}$ band considerably deviates from the values previously reported for silica films and single crystals, although it falls in the range obtained for the thinnest (1-3 ML) films thermally grown on $\mathrm{Si}(100)$.
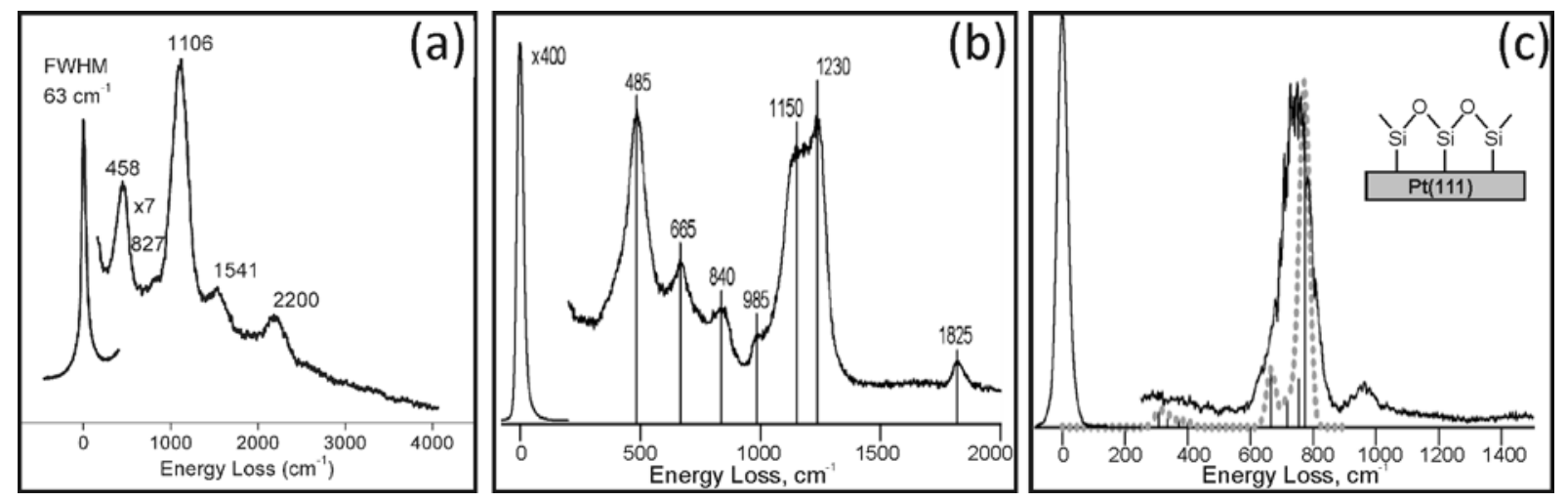

Figure 14. HREELS spectra of: (a) $2.8 \mathrm{~nm}$ thick $\mathrm{SiO}_{2}$ film grown on $\mathrm{Pd}(100)$. Reprinted from ref. ${ }^{[66]}$ Copyright (2008) with permission from Elsevier. (b) $\mathrm{SiO}_{\mathrm{x}}$ film grown on $\mathrm{Pd}(111)$ by chemical vapor deposition of $\mathrm{SiH}_{4}$ onto O-precovered surface, then annealed to $900 \mathrm{~K}$. Reprinted 
from ref. ${ }^{[67]}$ Copyright (2008) with permission from Elsevier. (c) $\mathrm{SiO}_{\mathrm{x}}$ film grown on $\mathrm{Pt}(111)$ by chemical vapor deposition of $\mathrm{SiH}_{4}$ onto O-precovered surface, then annealed to $800 \mathrm{~K}$. The dashed line represents the calculated spectrum for the chain-like structure schematically shown in the inset. Reprinted from ref. ${ }^{[68]}$ Copyright (2008) with permission from Elsevier.

STM images showed that the $\mathrm{SiO}_{2}$ film surface is uniform and atomically flat, and the terraces are notably free of voids and any other defects. A high-resolution image of the terraces revealed a square mesh with a lattice constant of $3.6 \pm 0.2 \AA$, which is close to $\sqrt{ } 2(\approx 1.4)$ times that of $\operatorname{Pd}(100)$. Interestingly, the terrace step height is measured to be $2.8 \pm 0.2 \AA$, i.e. also 1.4 times of that on $\operatorname{Pd}(100)$. Unfortunately, no LEED patterns were provided by the authors in order to justify a long-range ordering and symmetry of the silica overlayers. Finally, ISS measurements of the vacuum-annealed films indicated that Pd may diffuse to the silica surface even at $800 \mathrm{~K}$. This finding, therefore, limits the preparation to low temperatures only. All in all, the atomic structure of the films grown on $\operatorname{Pd}(100)$ cannot be regarded as established.

Silica structures on $\operatorname{Pd}(111)$ at sub-monolayer surface coverage were grown by Medlin and co-workers using coadsorption of $\mathrm{SiH}_{4}$ and oxygen. ${ }^{[67]} \mathrm{TPD}$, HREELS, and AES results indicated that surface $\mathrm{Si}$ and $\mathrm{O}$ react at $<200 \mathrm{~K}$ to form a surface $\mathrm{SiO}_{\mathrm{x}}$ layer that is stable to at least $950 \mathrm{~K}$. A characteristic HREELS spectrum of the annealed $\mathrm{SiO}_{\mathrm{x}}$ layer is shown in Fig. 14b. In accordance with data reported for different silica structures (see the previous section), the peak at $485 \mathrm{~cm}^{-1}$ was assigned to Si-O-Si bending modes, the peak at $665 \mathrm{~cm}^{-1}$ - to symmetric Si-O-Si stretching, and the peak at $1150 \mathrm{~cm}^{-1}$ to $\mathrm{Si}-\mathrm{O}-\mathrm{Si}$ asymmetric stretching. Along with Chen et al. ${ }^{[39]}$, the authors proposed that the vibration at $985 \mathrm{~cm}^{-1}$ (as a shoulder) is due to Si-OPd asymmetric stretching. The peak at $1230 \mathrm{~cm}^{-1}$ that dominated after high-temperature 
annealing was taken as evidence for the formation of a long-range network of $\mathrm{Si}-\mathrm{O}$ bonding at high temperatures.

Later, this group performed similar experiments with Pt(111). ${ }^{[68]}$ HREELS spectra after low exposures of $\mathrm{SiH}_{4}$ on an O-saturated $\mathrm{Pt}(111)$ surface consisted of three primary peaks at 480, 800 , and $1130 \mathrm{~cm}^{-1}$, similar to those modes observed in their experiments with $\operatorname{Pd}(111)$. However, spectra measured after higher exposures of $\mathrm{SiH}_{4}$ showed a single dominant peak at $\sim 750 \mathrm{~cm}^{-1}$ (Fig. 14c) indicating a different surface structure at higher Si coverage. The following-up DFT calculations ${ }^{[69]}$ for both $\operatorname{Pd}(111)$ and $\operatorname{Pt}(111)$ surfaces were focused on the low coverage regime, hence only isolated and chain-like $\mathrm{SiO}_{\mathrm{x}}$ structures were considered. A vibrational mode analysis showed that chain formation, as schematically shown in the inset of Fig. 14c, may explain the experimental results on $\operatorname{Pt}(111)$, but not on $\operatorname{Pd}(111) .{ }^{[67]}$

\subsection{Silica films on Ruthenium}

Very recently, our group has initiated studies on the growth of thin silica films on $\operatorname{Ru}(0001) .{ }^{[70]}$ This substrate was previously used successfully for the growth of crystalline $\mathrm{CeO}_{2}(111)$ films despite a very large ( 40\%) mismatch between ceria and Ru lattices. ${ }^{[71]}$ Among numerous preparation recipes tested, a low temperature $(\sim 100 \mathrm{~K}) \mathrm{Si}$ deposition onto the $3 \mathrm{O}(2 \times 2)-\mathrm{Ru}(0001)$ surface in $\sim 10^{-7} \mathrm{mbar}^{\mathrm{O}}$ and subsequent annealing at $\sim 1200 \mathrm{~K}$ in $\sim 10^{-6}$ mbar $\mathrm{O}_{2}$ showed the best quality. The presence of chemisorbed oxygen prevents intermixing of $\mathrm{Si}$ and $\mathrm{Ru}$, as judged by XPS, and supplies more reactive, i.e. atomic, oxygen species for oxidation of the Si deposits than molecular oxygen in the gas phase. A low substrate temperature during the Si deposition to $\sim 100 \mathrm{~K}$ most likely suppresses the diffusivity of Si atoms on the 
surface, thereby favoring the formation of two-dimensional structures prior to final oxidation at high temperatures. The full oxidation and film ordering occurs upon high temperature annealing in oxygen (ca. $10^{-6} \mathrm{mbar}$ ) at 1150 - $1200 \mathrm{~K}$, ultimately resulting in a (2×2) LEED pattern.

At a Si coverage of about $0.5 \mathrm{ML}$ (with respect to $\mathrm{Ru}(0001)$ ), the atomically flat surface expresses domains with a honeycomb-like structure with a $\sim 5.5 \AA$ periodicity. ${ }^{[72]}$ The domains are shifted by half a lattice constant with respect to each other, thus producing a network of domain boundaries (Fig. 15a). The silica film is $\sim 1.4 \AA$ in apparent height with respect to underlying support. The respective IRAS spectrum shows the principal band at $1134 \mathrm{~cm}^{-1}$ and weaker signals at 1074, 790 and $687 \mathrm{~cm}^{-1}$ (Fig. 15b), which clearly resemble those observed for $\mathrm{SiO}_{2.5} / \mathrm{Mo}(112)$ films (see Fig. 9). XPS spectra of the film are also very similar to that of the Mo(112) supported film, where the O1s core level revealed two components centered at 531.3 and $529.8 \mathrm{eV}$ with a similar peak separation $(1.5 \mathrm{eV})$ and the intensity ratio $(\sim 3: 2)$ as for $\mathrm{SiO}_{2.5} / \mathrm{Mo}(112)$. Given the STM results, showing the formation of a complete overlayer with $(2 \times 2)$ lattice structure, and the agreement between XPS and IRAS results for this film with those previously reported for $\mathrm{SiO}_{2.5} / \mathrm{Mo}(112),{ }^{[45 a, 48]}$ a similar structure for silica films over $\mathrm{Ru}(0001)$ is suggested under these conditions. Namely, the film is formed by a honeycomb shaped network of tetrahedral Si-O linkages with a $5.4 \AA$ lattice constant, in which every Si forms one Si-O-Ru bond and three bridging Si-O-Si bonds. (For simplicity, the Si coverage is henceforth presented in monolayers equivalent (MLE), such that 1 MLE corresponds to a closed monolayer film). 

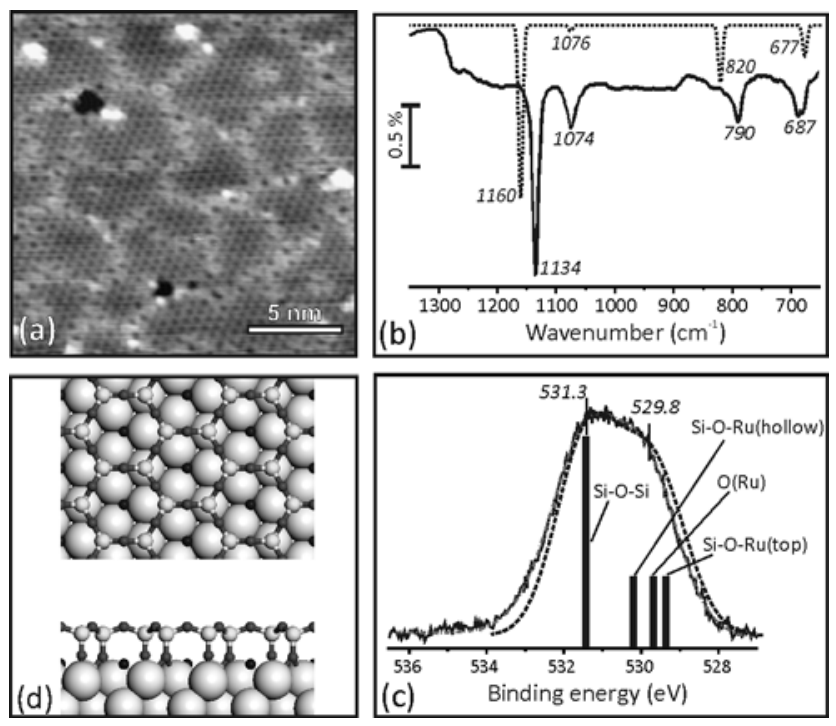

Figure 15. STM image (a), IRAS (b) and O1s XPS (c) spectra of a $\mathrm{SiO}_{2.5} / \mathrm{Ru}(0001)$ film. Dashed lines show the IRAS and XPS spectra, computed for the structural model, shown in (d), and artificially broadened to fit the experimental spectra. (O: small black; Si: small gray; Ru: large gray).

DFT calculations nicely corroborated the proposed structure. ${ }^{[72]}$ It has turned out, however, that among the several monolayer structures computed, only the film containing additional oxygen atoms directly adsorbed on $\mathrm{Ru}$ (henceforth referred to as $\mathrm{O}(\mathrm{Ru})$ atoms) is stable (Fig. 15d). The harmonic IRA spectrum (see Fig. 15c) simulated for the most stable monolayer structure revealed the most intense IR-active mode at $\sim 1160 \mathrm{~cm}^{-1}$, which originates from the in-phase combination of asymmetric stretching vibrations of the Si-O-Ru linkages. The much weaker mode at $1076 \mathrm{~cm}^{-1}$ involves combinations of symmetric O-Si-O stretching vibrations. The bands at 820 and $677 \mathrm{~cm}^{-1}$ represent the combinations of asymmetric stretching of Si-O-Ru linkages and O-Si-O bending modes. The positions and relative intensities of those 
calculated bands are in good agreement with the experimental data, as is the XPS spectrum simulated for this structure (Fig. 15c).
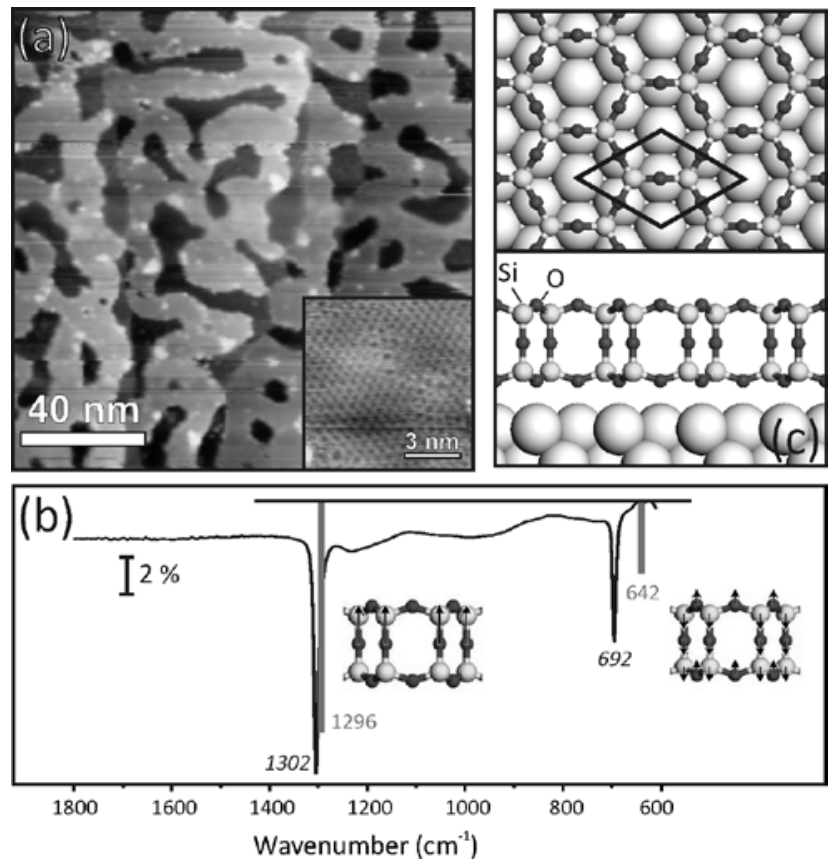

Figure 16. Large-scale (a) and high-resolution (inset) STM images and (b) IRAS spectrum of a $\mathrm{SiO}_{2} / \mathrm{Ru}(0001)$ film. IRA-active vibrational modes in the structural model (c) are shown as bars with their height proportional to the intensity. The "O-poor" structure shown in (c) may contain additional oxygen atoms chemisorbed only to the Ru atoms, thus forming "O-rich" phases. The $(2 \times 2)$ unit cell is indicated.

Increasing Si coverage up to 2 MLE resulted in no change in the surface symmetry, still showing a $(2 \times 2)$ LEED pattern. However, the IRAS spectra revealed very sharp (thus indicating good crystallinity) and intense bands at 1300 and $692 \mathrm{~cm}^{-1}$ which developed with concomitant disappearance of the bands associated with the monolayer structure (Fig. 16b). STM inspection 
of the prepared films showed atomically flat morphology, exposing some holes of $\sim 5 \AA$ in apparent depth. High-resolution STM images of such structures showed again a honeycomb-like network with a $~ 5.5 \AA$ periodicity (inset in Fig. 16a), similar to the monolayer films (Fig. 15a). Analysis of different structures previously considered in DFT calculations of silica films on $\operatorname{Mo}(112)^{[47 a]}$ led to the suggestion that, in this case, a "bilayer" structure composed of two monolayers linked together through bridging $\mathrm{O}$ atoms is formed as shown in Fig. 16c,. Since this structure can also be obtained in two deposition-oxidation steps with 1 MLE Si each, the transformation of the mono- into the bi-layer structure must be accompanied by breaking Si-ORu bonds and creating the Si-O-Si linkages. Apparently, such a process is a thermodynamically unfavorable in the case of the Mo(112) support. As a result, the formation of bilayer films on Mo(112) has never been observed.

The bilayer model was examined by DFT including semi-empirical dispersion correction $(\mathrm{DFT}+\mathrm{D}) .{ }^{[70]}$ The calculated adhesion energy of the bilayer silica film to the $\mathrm{Ru}(0001)$ support was only about $3 \mathrm{~kJ} \mathrm{~mol}^{-1} \AA^{-2}$, with the main contribution coming from the dispersion term. The phonon spectrum, calculated for this structure, revealed two IR active modes (see Fig. 16b). The most intense mode at $1296 \mathrm{~cm}^{-1}$ represents an in-phase combination of asymmetric Si-O-Si stretching vibrations of the Si-O-Si linkage between two layers. The second mode at $642 \mathrm{~cm}^{-1}$ is a combination of symmetric Si-O-Si stretching vibrations of Si-O-Si bonds nearly parallel to the surface. Not only the positions but also the relative intensities of the vibrational bands are in very good agreement with the experimental results.

Combined XPS/UPS and DFT studies ${ }^{[73]}$ further showed that the system may exist in "Opoor" and "O-rich" configurations, depending on the amount of $\mathrm{O}(\mathrm{Ru})$ atoms directly chemisorbed onto the $\mathrm{Ru}(0001)$ surface. The presence of the $\mathrm{O}(\mathrm{Ru})$ atoms considerably lowers 
the adhesion energy. Almost certainly, this effect is connected to the repulsive interaction between the oxygen atoms of the silica film and the $\mathrm{O}(\mathrm{Ru})$ atoms. As a result the distance between the silica sheet and metal support increases. The $\mathrm{SiO}_{2} / \mathrm{Ru}$ electronic states reversibly vary with the amounts of adsorbed oxygen underneath the silica film. In principle, this opens the possibility to tune the electronic properties of oxide/metal systems without altering the thickness and structure of an oxide overlayer itself as discussed before for the monolayer silica films on $\operatorname{Mo}(112){ }^{[74]}$ Interestingly, the transition between the O-poor and O-rich states is fully reversible via annealing and reoxidation treatments, whereas the O-rich films are much more stable in the case of $\mathrm{SiO}_{2.5} / \mathrm{Mo}(112)$, since the O-Mo bond is much stronger than the O-Ru bond.

In order to see whether the silica film on $\mathrm{Ru}(0001)$ can be further grown in a layer-bylayer mode, thicker silica films were prepared by deposition of 4 MLE Si. ${ }^{[72]}$ The results were almost independent of whether the films were prepared in one step or in two sequential 2 MLE deposition-oxidation steps. STM images of the films revealed a smooth surface, albeit not atomically flat. Unfortunately, attempts to achieve atomic resolution were not successful, as an STM imaging becomes unstable for thick insulating films. As in the case of mono- and bilayer structures, the substantial changes are observed in IRAS spectra: The intensities of the 1300 and $694 \mathrm{~cm}^{-1}$ peaks strongly decreased, and a new band developed at $1257 \mathrm{~cm}^{-1}$ with a prominent shoulder at $1164 \mathrm{~cm}^{-1}$. The shape and position of this band are characteristic for the LO vibration modes in bulk-like silica (see Figs. 3 and 5). It therefore appears that thicker films exhibit a three-dimensional network of $\left[\mathrm{SiO}_{4}\right]$ tetrahedra rather than the layered structure observed for mono- and bi-layer films. In this case, the termination of the film may be ill-defined, resulting in relatively high surface corrugation (ca. $1 \AA$ ) as measured by STM. 


\subsection{Silica films on Platinum}

In order to elucidate the role of the metal support in the films' atomic structure, silica films were grown on Pt(111) for comparison. ${ }^{[75]}$ The Pt(111) surface has a slightly larger lattice constant, than $\mathrm{Ru}(0001)$, i.e., 2.77 and $2.71 \AA$, respectively. In addition, the film preparations on Mo(112) and Ru(0001) include oxygen precovered surfaces prior to the Si deposition in the oxygen ambient. In this respect, Pt as a noble metal may behave differently than the Mo and Ru supports

The film preparation was basically the same as that on $\mathrm{Ru}(0001)$. Upon Si deposition, a new Pt 3d state was observed by XPS as a shoulder at low binding energy, thus indicating Pt-Si surface intermixing, which was not observed on a Ru support most likely due to the presence of a $30(2 \times 2)$ ad-layer that oxidizes the first incoming $\mathrm{Si}$ atoms. This state disappeared upon subsequent oxidation at high temperatures. The XPS spectra of the finally prepared films were very similar to those obtained for the bilayer film on $\mathrm{Ru}(0001)$, except that the $\sim 530 \mathrm{eV}$ signal for the O1s level (see e.g. Fig. 15c) was less prominent on Pt(111). Since this signal is associated with oxygen atoms directly adsorbed on the metal surface underneath a film and/or in holes exposing a metal support in the not fully covered films, the difference can easily be attributed to the lower affinity of $\mathrm{Pt}(111)$ toward oxygen as compared to $\mathrm{Ru}(0001)$.

The IRAS spectra of the 1 and 2 MLE films revealed the bilayer structure of the resulting film with sharp bands at 1294 and $690 \mathrm{~cm}^{-1}$ exclusively (Fig. 17a). The band intensity scales with the Si coverage and does not show any feature at $1000-1100 \mathrm{~cm}^{-1}$ otherwise expected for monolayer films. This scaling behavior is also observed in XPS spectra, although the O/Pt(111) signal at $~ 530 \mathrm{eV}$ becomes more pronounced for a 1 MLE film since the sample must expose a 
larger fraction of the Si-free, O-covered Pt surface. The results, therefore, indicate the formation of exclusively bilayer films under the conditions applied. The respective STM images (Figs. 17(b-d) do not reveal an ordered, crystalline overlayer, but rather the formation of a twodimensional vitreous silica film (see details in the next section).

The apparent thickness of the bilayer silica films on $\operatorname{Pt}(111)$, i.e. on the order of $2 \AA$, is considerably lower than $\sim 5 \AA$ measured for the bilayer films on $\mathrm{Ru}(0001)$, which is in turn very close to the geometrical film thickness determined by DFT. Such behavior can be assigned to the electronic effects frequently observed for STM imaging of metal-oxide systems, which, in addition, may be bias- and polarity-dependent. As a side note, this finding again points to the general conclusion that only a multi-technique approach allows for determination of the atomic structure of metal supported thin oxide films.
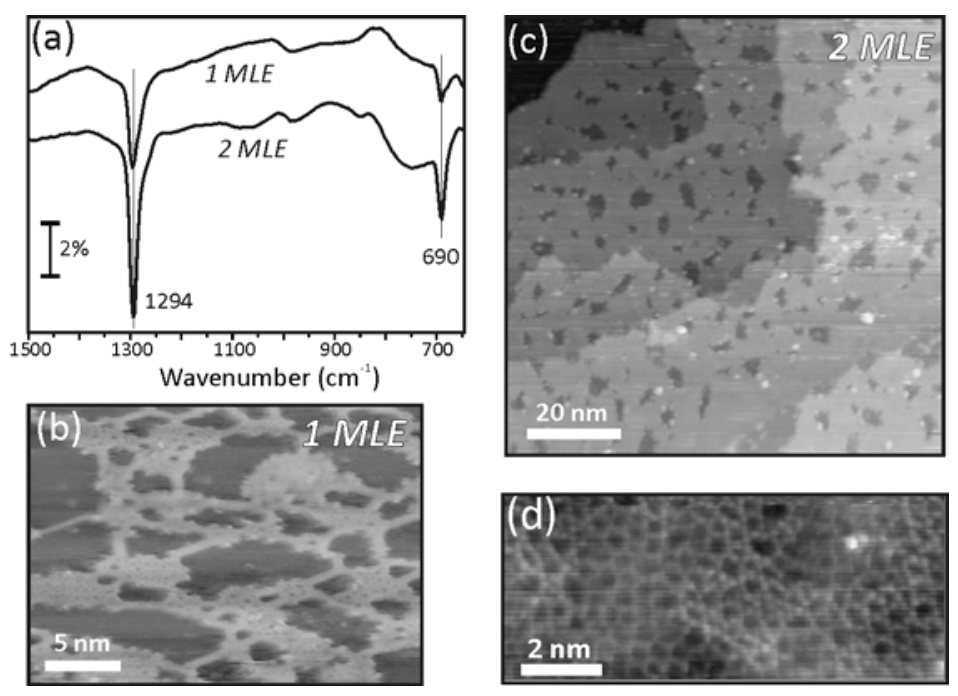

Figure 17. IRAS spectra (a) and STM images (b,c) of $\mathrm{SiO}_{2}$ films grown on $\mathrm{Pt}(111)$ at 1 and 2 MLE coverages as indicated. (d) High resolution STM image of a (vitreous) silica bilayer observed at both coverages. 
Interestingly, the silica islands formed at sub-monolayer coverages on $\operatorname{Pt}(111)$ are bridged by narrow stripes of the same height running primarily along the principal crystallographic directions of Pt(111). Most of these "bridges" are $\sim 7 \AA$ in width, although $\sim 4 \AA$ and $\sim 10 \AA$ wide lines were observed as well. To some extent, these stripes resemble onedimensional silica rows formed at low Si coverage on Mo(112) (see Fig. 13), although its atomic structure has not yet be identified.

\subsection{Crystalline and vitreous films}

In the course of preparation of the bilayer silica films on $\mathrm{Ru}(0001)$ it was realized that LEED patterns sometimes show both $(2 \times 2)$ spots and a diffraction ring representing the same lattice constant. On Pt(111), only a (2×2) diffraction ring was observed under the conditions studied. The diffraction rings are characteristic for randomly oriented crystallites such as those observed in powders, which could be identified as vitreous (amorphous, glassy) silica.

One of the first proposed theories of glasses, the so-called "crystallite" theory envisaged glass as an aggregate of extremely small crystals (“crystallites”) with fairly sharp external boundaries, which are connected with each other in a random way. (Although it is generally attributed to Lebedev's paper published in 1921, it appears that the crystallite theory was in fact

first mentioned in 1835 by Frankenheim). ${ }^{[3]}$ Later, in his famous paper in 1932, Zachariasen ${ }^{[76]}$ proposed that the glass consists of a three-dimensional random network (RN) of tetrahedral $\mathrm{SiO}_{4}$ molecular units. One of the first x-ray diffraction (XRD) studies of silica glasses by Warren ${ }^{[77]}$ 
favored the RN model, which accounts satisfactorily for the observed x-ray as well as neutron radial distribution functions of vitreous silica. ${ }^{[3,78]}$ Neighboring $\mathrm{SiO}_{4}$ units in the solid are linked at the vertices by sharing a common or bridging oxygen atom. Although the $\mathrm{SiO}_{4}$ units themselves are thought to be effectively regular in shape, the mutual orientation of adjacent units can vary widely throughout the glass in a manner which is apparently random in character, subject only to the restriction that the network be continuously connected. There were several attempts to find a compromise between the two models, which differ essentially in the size of the short-range ordering (15-20 $\AA$ in the crystallite theory versus $7-8 \AA$ in the RN theory). For example, Görlich et al. ${ }^{[79]}$ proposed the "sintered powder model”, where vitreous silica forms a loose and randomly mixed texture of high-temperature $\alpha$-cristobalite; between the domains there exists disorder. Another model includes a continuous random network, but of deformed sixmembered rings (see, for example, ref. ${ }^{[5]}$ ) These models are schematically depicted in Fig. 18.

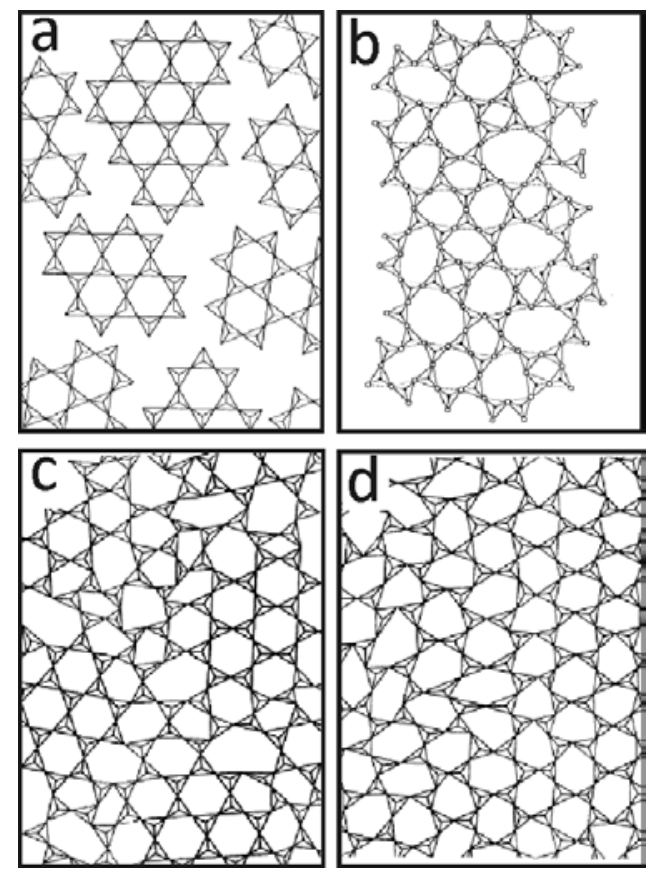


Figure 18. Schematic representations of structural models for vitreous silica: (a) crystallite; (b) random network; (c) ordered domains; (d) distorted network.

Nonetheless, none of these models was justified with any imaging (microscopy) technique. Although modern transmission and scanning electron microscopes possess atomic resolution (particularly when aberration correction is applied) to resolve atomic spacings in disordered systems, these techniques commonly produce images which are 2D projections of 3D structures. In this context, the thin films, which are well-suited materials for STM and AFM, can be employed to directly visualize the atomic structure of the amorphous silica as has been recently demonstrated in our own laboratories. ${ }^{[75,80]}$
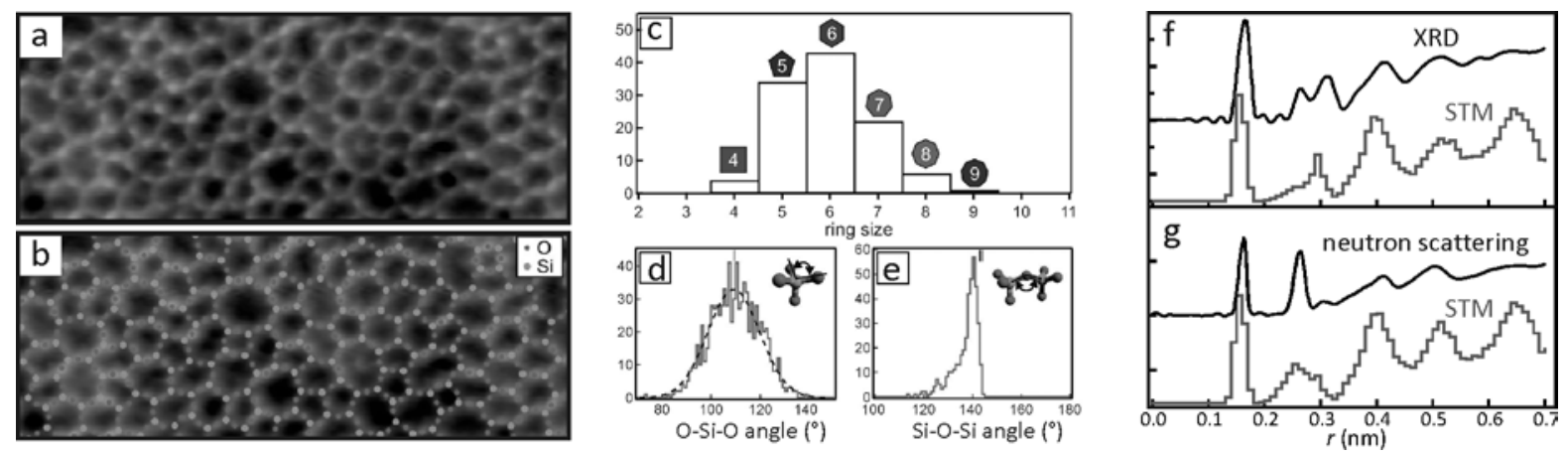

Figure 19. (a) High resolution STM image $(8 \mathrm{~nm} \times 3 \mathrm{~nm})$ of an amorphous silica film on $\mathrm{Ru}(0001)$. (b) The same image superimposed with a model, where $\mathrm{O}$ atoms are imaged as protrusions forming $\mathrm{N}$-membered rings $(\mathrm{N}=4-8)$. (c-e) The ring size and angle distributions in the silica film derived from STM images. (f,g) Pair correlation function obtained by STM in comparison with X-ray and neutron radial distribution factors of vitreous silica. ${ }^{[80]}$ 
Figure 19a shows an atomically resolved STM image of a disordered silica bilayer film on $\mathrm{Ru}(0001) .{ }^{[80]}$ The polygonal network in the surface plane can be clearly recognized. The protrusions are grouped in triangles, and as such they may be assigned to three of four $\mathrm{O}$ atoms in the $\mathrm{SiO}_{4}$ units. The position of the $\mathrm{Si}$ atoms may then be calculated. Figure 19b displays the same STM image superimposed with the positions of the $\mathrm{Si}$ and $\mathrm{O}$ atoms. A histogram revealed that the ring size, measured as a number $(\mathrm{N})$ of members forming the ring, varies between $\mathrm{N}=4$ and 9, with a maximum at $\mathrm{N}=6$ that corresponds to the pure crystalline structure (Fig. 19c). The intra-tetrahedral Si-O-Si angle (Fig. 19d) showed a symmetric distribution with an average of $110^{\circ}\left(+-10^{\circ}\right)$ which fits well the $109.5^{\circ}$ angle in a regular tetrahedron. The histogram of the SiO-Si angles (Fig. 19e) revealed a peak at $141^{\circ}$, which is, again, in nice agreement to the average angle of $143^{\circ}$ obtained for vitreous silica by ${ }^{29} \mathrm{Si}$ magic-angle spinning nuclear magnetic resonance spectroscopy ${ }^{[81]}$ and $144^{\circ}$ by XRD. ${ }^{[82]}$ A sharp edge at $145^{\circ}$ manifests the flat and 2D character of the silica film. (Note, that in this analysis the structural information about the zdirection (normal to the surface) was taken from the previous DFT calculations of the crystalline bilayer film, see Fig. 16). ${ }^{[70]}$

From the STM images, the pair correlation function was obtained using X-ray and neutron scattering factors of $\mathrm{Si}$ and $\mathrm{O}$ according to the formula from refs. ${ }^{[78,83]}$, which were normalized by $r^{-1}$ to account for the 2D structure of the film. The peak positions and their relative intensities are shown in Figs. 19(f,g) which reveal good agreement with those obtained by XRD and neutron scattering. The small deviations are not surprising since the silica bilayer film on $\mathrm{Ru}(0001)$ is atomically flat in contrast to 3D silica glasses used for comparison. 


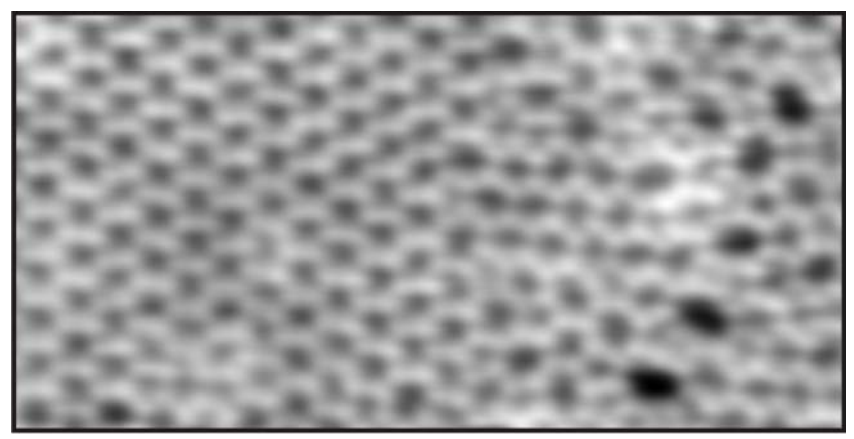

Figure 20. High resolution STM image $(10 \mathrm{~nm} \times 5 \mathrm{~nm})$ of a silica bilayer film, showing crystalline (left portion) and vitreous (right portion) phases coexisting.

Furthermore, direct STM imaging of the bilayer silica films allows one to address structural transformation between crystalline and vitreous phases. ${ }^{[84]}$ Figure 20 displays an STM image, where the crystalline phase smoothly transforms into the vitreous one without any "defects" in terms of unsaturated bonds or different bonding arrangements beyond cornersharing $\left[\mathrm{SiO}_{4}\right]$ tetrahedra. The transition region extends no more than $1.5 \mathrm{~nm}$, in which two adjacent 6-membered rings "disproportionate" into the 5- and 7-membered rings until the distribution of a pure vitreous ring network is reached.

Analysis of bilayer films prepared under different conditions by varying annealing time, temperature, oxygen pressure, etc. showed that the rate of sample cooling after the hightemperature oxidation step may play the key role in controlling crystallinity of the films on $\mathrm{Ru}(0001)$. Note also, that once formed, the vitreous film, prepared by fast cooling, cannot be transformed into the crystalline state by re-oxidation of the same sample followed by slow cooling, and vice versa. Applying higher temperatures leads to film decomposition. These findings agree well with the very high barrier for crystal-to-glassy transformation predicted by the DFT calculations. ${ }^{[80]}$ The influence of the cooling rate has been examined also for the 
monolayer silica films on $\mathrm{Ru}(0001)$ that showed essentially no effect. Most likely, the strong SiO-Ru bonds drive the monolayer film to be in registry with a $\mathrm{Ru}(0001)$ substrate, ultimately resulting in the long-range ordering of the films.

Very recently Huang et al. ${ }^{[85]}$ reported TEM and STEM studies of ultrathin silica films accidently synthesized during the chemical vapor deposition growth of graphene. Image analysis showed close similarities to the results of Lichtenstein et al. ${ }^{[80]}$ Both studies demonstrated that bilayer silica films, weakly bound to an underlying support, provide the unique possibility to study silicate glasses and the glass transition with atomic precision in real space and, eventually, in time, for example, employing low energy electron microscopy.

\subsection{The support effects: General trend}

We are now in position to compare the structures of ultra-thin silica films formed on three different supports (Mo, Ru, Pt). The principal structure of the silica films can be identified by IRA spectroscopy, as each structure shows a characteristic vibrational band, i.e., $\sim 1100 \mathrm{~cm}^{-1}$ for monolayer films, $\sim 1300 \mathrm{~cm}^{-1}$ for the bilayer structures, and $\sim 1250 \mathrm{~cm}^{-1}$ for the bulk-like silica films.

On Mo(112), silica grows only as a monolayer strongly bonded to Mo via the Si-O-Mo linkages. On Ru(0001), both monolayer, bonded through the Si-O-Ru linkage, and bilayer films, weakly bonded to $\mathrm{Ru}$, are observed, depending on the $\mathrm{Si}$ coverage. Irrespective of the $\mathrm{Si}$ coverage, only bilayer films grew on $\operatorname{Pt}(111)$. There is an obvious trend in the principal structure which correlates with the oxygen affinity to the metal support. Indeed, heats of dissociative adsorption of oxygen are of $544,-220$, and $-133 \mathrm{~kJ} / \mathrm{mole}$ for Mo, Ru, and Pt, respectively. 
Therefore, the SiO-Mo bond is much stronger than the SiO-Pt bond, and it will favor the monolayer structure, whereas on $\mathrm{Pt}(111)$ silica forms a "closed shell" bilayer structure terminated by the fully saturated oxygen layer on either side. $\mathrm{Ru}(0001)$ exhibits intermediate properties and allows formation of both mono- and bi-layer structures.

Another issue relates to the role of the lattice mismatch between a silica film and a metal substrate, which is obviously different for the three metals studied. The lattice constant of the unsupported, free-standing silica bilayer is computed to be $5.24-5.32 \AA^{[45 b, 59]}$. When supported on $\mathrm{Ru}(0001)$ (lattice constant $2.71 \AA$ ) and $\operatorname{Pt}(111)$ (lattice constant $2.77 \AA$ ), the most "natural” structure for the silica film to accommodate these metal supports is the $(2 \times 2)$ structure, resulting in a periodicity of 5.42 and $5.54 \AA$, respectively, that is indeed observed by LEED and STM. From this point of view, $\mathrm{Ru}(0001)$ is better suited than $\mathrm{Pt}(111)$ as the lattice mismatch is smaller. In principle, this may explain why only amorphous films were so far obtained on $\operatorname{Pt}(111)$.

\section{Summary and concluding remarks}

Based on the results available to date, one may safely conclude that ultrathin silica films on $\mathrm{Mo(112),} \mathrm{Ru}(0001)$ and, to lesser extent, on $\mathrm{Pt}(111)$ seem to be the only silica films whose structures are established at the atomic level. The results show that the metal-oxygen bond strength plays the decisive role. Metals with high oxygen adsorption energy favor the formation of crystalline films as a monolayer network of corner-sharing $\left[\mathrm{SiO}_{4}\right]$ tetrahedra with $\mathrm{SiO}_{2.5}$ composition. Noble metals primarily form bilayer $\mathrm{SiO}_{2}$ sheets. The metals with intermediate energies may form either of the structures. In contrast to monolayer films, the bilayer films exist in both crystalline and amorphous forms. These two-dimensional silica films provide unique 
opportunities to directly visualize the atomic structure of vitreous silica. The results open a new playground for studying the fundamental aspects of glasses such as the order-disorder transition. In addition, such systems also open up opportunities to look for model systems involving silica films in the area of catalysis, e.g. as a support anchoring specific metal species. Finally, doping these silica films with $\mathrm{Al}$ allowed preparation of aluminosilicate films which can be considered as adequate model systems for zeolite surfaces. ${ }^{[86]}$

The results reveal a structural complexity and diversity of the silica overlayers on metals and provide further steps towards our understanding of the atomic structure, structural dynamics and physical and chemical properties of silica.

\section{Acknowledgements}

We thank all our co-workers, whose names appear in the cited papers, for their tremendous work in the laboratories. We acknowledge collaboration with the groups of J. Sauer (Humboldt University in Berlin) and G. Pacchioni (University Milano) as well as fruitful discussions with D.W. Goodman and his coworkers (Texas A \& M University). This work has been supported by Fonds der Chemischen Industrie and Deutsche Forschungsgemeinschaft. 


\section{References}

[1] D. A. Muller, T. Sorsch, S. Moccio, F. H. Baumann, K. Evans-Lutterodt, G. Timp, Nature 1999, 399, 758-761.

[2] J. T. Yates Jr, Surface Science 2004, 565, 103-106.

[3] A. C. Wright, Journal of Non-Crystalline Solids 1994, 179, 84-115.

[4] A. K. A. Pryde, M. T. Dove, Physics and Chemistry of Minerals 1998, 26, 171-179.

[5] D. Keen, M. Dove, Journal of Physics: Condensed Matter 1999, 11, 9263.

[6] a) C. R. Helms, E. H. Poindexter, Reports on Progress in Physics 1994, 57, 791; b) K. Hirose, H. Nohira, K. Azuma, T. Hattori, Progress in Surface Science 2007, 82, 3-54.

[7] J. Derrie, M. Commandré, Surface Science 1982, 118, 32-46.

[8] N. Cabrera, N. F. Mott, Rep. Prog. Phys. 1948, 12, 163.

[9] a) J. A. Schaefer, W. Göpel, Surface Science 1985, 155, 535-552; b) H. Ibach, H. D. Bruchmann, H. Wagner, Applied Physics A Solids and Surfaces 1982, 29, 113-124.

[10] P. A. Thiry, M. Liehr, J. J. Pireaux, R. Sporken, R. Caudano, J. P. Vigneron, A. A. Lucas, Journal of Vacuum Science \& Technology B: Microelectronics and Nanometer Structures 1985, 3, 1118-1121.

[11] J. Finster, D. Schulze, F. Bechstedt, A. Meisel, Surface Science 1985, 152-153, 10631070.

[12] P. J. Grunthaner, M. H. Hecht, F. J. Grunthaner, N. M. Johnson, Journal of Applied Physics 1987, 61, 629-638.

[13] W. Braun, H. Kuhlenbeck, Surface Science 1987, 180, 279-288. 
[14] S. Ichimura, A. Kurokawa, K. Nakamura, H. Itoh, H. Nonaka, K. Koike, Thin Solid Films 2000, 377-378, 518-524.

[15] a) J. M. Shaw, N. Herbots, Q. B. Hurst, D. Bradley, R. J. Culbertson, V. Atluri, K. T. Queeney, Journal of Applied Physics 2006, 100, 104109-104104; b) N. Herbots, J. M. Shaw, Q. B. Hurst, M. P. Grams, R. J. Culbertson, D. J. Smith, V. Atluri, P. Zimmerman, K. T. Queeney, Materials Science and Engineering B: Solid-State Materials for Advanced Technology 2001, 87, 303-316.

[16] N. Herbots, J. M. Shaw, Q. B. Hurst, M. P. Grams, R. J. Culbertson, D. J. Smith, V. Atluri, P. Zimmerman, K. T. Queeney, Materials Science and Engineering: B 2001, 87, 303-316.

[17] C. T. Kirk, Physical Review B 1988, 38, 1255-1273.

[18] D. W. Berreman, Physical Review 1963, 130, 2193-2198.

[19] K. T. Queeney, M. K. Weldon, J. P. Chang, Y. J. Chabal, A. B. Gurevich, J. Sapjeta, R. L. Opila, Journal of Applied Physics 2000, 87, 1322-1330.

[20] F. Giustino, A. Pasquarello, Physical Review Letters 2005, 95, 187402.

[21] K. T. Queeney, Y. J. Chabal, M. K. Weldon, K. Raghavachari, Physica status solidi (a) 1999, 175, 77-88.

[22] G. Feliciano, B. Angelo, P. Alfredo, Journal of Physics: Condensed Matter 2005, 17, S2065.

[23] C. Raynaud, Journal of Non-Crystalline Solids 2001, 280, 1-31.

[24] U. Starke, J. Schardt, J. Bernhardt, K. Heinz, Journal of Vacuum Science and Technology A 1999, 17, 1688-1692. 
[25] J. Bernhardt, J. Schardt, U. Starke, K. Heinz, Applied Physics Letters 1999, 74, 10841086.

[26] W. Lu, P. Krüger, J. Pollmann, Physical Review B 2000, 61, 13737-13744.

[27] M. Hollering, F. Maier, N. Sieber, M. Stammler, J. Ristein, L. Ley, A. P. J. Stampfl, J. D. Riley, R. C. G. Leckey, F. P. Leisenberger, F. P. Netzer, Surface Science 1999, 442, 531542.

[28] B. Hornetz, H. J. Michel, J. Halbritter, J. Mater. Res. 1994, 9, 3088-3094.

[29] H. Tsuchida, I. Kamata, K. Izumi, Applied Surface Science 1997, 117-118, 225-229.

[30] a) H. P. Bonzel, A. M. Franken, G. Pirug, Surface Science 1981, 104, 625-642; b) P. Légaré, L. Hilaire, G. Maire, Surface Science 1984, 141, 604-616.

[31] K. E. Keck, B. Kasemo, Surface Science 1986, 167, 313-330.

[32] C. S. Ko, R. J. Gorte, Surface Science 1985, 155, 296-312.

[33] a) X. Xu, D. W. Goodman, Applied Physics Letters 1992, 61, 774-776; b) X. Xu, D. W. Goodman, Surface Science 1993, 282, 323-332.

[34] K. Luo, D. Y. Kim, D. W. Goodman, Journal of Molecular Catalysis A: Chemical 2001, $167,191-198$.

[35] J. W. He, X. Xu, J. S. Corneille, D. W. Goodman, Surface Science 1992, 279, 119-126.

[36] T. Schroeder, PhD Thesis 2001.

[37] a) T. Schroeder, M. Adelt, B. Richter, M. Naschitzki, M. Baumer, H. J. Freund, Surface Review and Letters 2000, 7, 7-14; b) T. Schroeder, M. Adelt, B. Richter, M. Naschitzki, M. Bäumer, H. J. Freund, Microelectronics Reliability 2000, 40, 841-844; c) T. Schroeder, A. Hammoudeh, M. Pykavy, N. Magg, M. Adelt, M. Bäumer, H. J. Freund, 
Solid-State Electronics 2001, 45, 1471-1478; d) T. Schroeder, J. B. Giorgi, M. Bäumer, H. J. Freund, Physical Review B 2002, 66, 165422.

[38] a) Y. D. Kim, T. Wei, D. W. Goodman, Langmuir 2002, 19, 354-357; b) S. Wendt, Y. D. Kim, D. W. Goodman, Progress in Surface Science 2003, 74, 141-159; c) Y. D. Kim, J. Stultz, T. Wei, D. W. Goodman, The Journal of Physical Chemistry B 2002, 107, 592596.

[39] M. S. Chen, A. K. Santra, D. W. Goodman, Physical Review B 2004, 69, 155404.

[40] D. Ricci, G. Pacchioni, Physical Review B 2004, 69, 161307.

[41] B. K. Min, W. T. Wallace, D. W. Goodman, The Journal of Physical Chemistry B 2004, $108,14609-14615$.

[42] A. K. Santra, B. K. Min, D. W. Goodman, Surface Science 2002, 515, L475-L479.

[43] E. Ozensoy, B. K. Min, A. K. Santra, D. W. Goodman, The Journal of Physical Chemistry B 2004, 108, 4351-4357.

[44] S. Wendt, E. Ozensoy, T. Wei, M. Frerichs, Y. Cai, M. S. Chen, D. W. Goodman, Physical Review B 2005, 72, 115409.

[45] a) J. Weissenrieder, S. Kaya, J. L. Lu, H. J. Gao, S. Shaikhutdinov, H. J. Freund, M. Sierka, T. K. Todorova, J. Sauer, Physical Review Letters 2005, 95, 076103; b) L. Giordano, D. Ricci, G. Pacchioni, P. Ugliengo, Surface Science 2005, 584, 225-236.

[46] S. Kaya, J. Weissenrieder, D. Stacchiola, T. K. Todorova, M. Sierka, J. Sauer, S. Shaikhutdinov, H. J. Freund, Surface Science 2008, 602, 3338-3342.

[47] a) T. K. Todorova, M. Sierka, J. Sauer, S. Kaya, J. Weissenrieder, J. L. Lu, H. J. Gao, S. Shaikhutdinov, H. J. Freund, Physical Review B 2006, 73, 165414; b) T. K. Todorova, M. 
Sierka, J. Sauer, S. Kaya, J. Weissenrieder, J. L. Lu, H. J. Gao, S. Shaikhutdinov, H. J. Freund, Physical Review B 2006, 73, 249902.

[48] S. Kaya, M. Baron, D. Stacchiola, J. Weissenrieder, S. Shaikhutdinov, T. K. Todorova, M. Sierka, J. Sauer, H. J. Freund, Surface Science 2007, 601, 4849-4861.

[49] T. K. Todorova, M. Sierka, J. Sauer, S. Kaya, J. Weissenrieder, J. L. Lu, H. J. Gao, S. Shaikhutdinov, H. J. Freund, Physical Review B 2006, 73.

[50] M. Sierka, Progress in Surface Science 2010, 85, 398-434.

[51] M. Sierka, T. K. Todorova, S. Kaya, D. Stacchiola, J. Weissenrieder, J. Lu, H. Gao, S. Shaikhutdinov, H.-J. Freund, J. Sauer, Chemical Physics Letters 2006, 424, 115-119.

[52] I. N. Yakovkin, Surface Review and Letters 2005, 12, 449-456.

[53] M. Chen, D. W. Goodman, Surface Science 2006, 600, L255-L259.

[54] J. L. Lu, S. Kaya, J. Weissenrieder, T. K. Todorova, M. Sierka, J. Sauer, H. J. Gao, S. Shaikhutdinov, H. J. Freund, Surface Science 2006, 600, L164-L168.

[55] a) L. Giordano, D. Ricci, G. Pacchioni, P. Ugliengo, Surface Science 2007, 601, 588-590;

b) M. Chen, D. W. Goodman, Surface Science 2007, 601, 591-593.

[56] a) J. Seifert, D. Blauth, H. Winter, Physical Review Letters 2009, 103, 017601; b) J. Seifert, M. Busch, A. Schüller, D. Blauth, S. Wethekam, H. Winter, Surface and Interface Analysis 2010, 42, 1575-1580; c) J. Seifert, A. Schüller, H. Winter, R. Włodarczyk, J. Sauer, M. Sierka, Physical Review B 2010, 82, 035436; d) J. Seifert, H. Winter, Surface Science 2009, 603, L109-L112.

[57] T. Kinoshita, S. Mizuno, Surface Science 2011, 605, 1209-1213.

[58] G. M. Rignanese, A. De Vita, J. C. Charlier, X. Gonze, R. Car, Physical Review B 2000, $61,13250-13255$. 
[59] C. Freysoldt, P. Rinke, M. Scheffler, Physical Review Letters 2007, 99, 086101.

[60] U. Martinez, L. Giordano, G. Pacchioni, The Journal of Physical Chemistry B 2006, 110, 17015-17023.

[61] S. Wendt, M. Frerichs, T. Wei, M. S. Chen, V. Kempter, D. W. Goodman, Surface Science 2004, 565, 107-120.

[62] S. Kaya, J. Weissenrieder, D. Stacchiola, S. Shaikhutdinov, H. J. Freund, The Journal of Physical Chemistry C 2006, 111, 759-764.

[63] J. Yang, E. G. Wang, Physical Review B 2006, 73, 035406.

[64] D. J. Stacchiola, M. Baron, S. Kaya, J. Weissenrieder, S. Shaikhutdinov, H. J. Freund, J. Freund, Applied Physics Letters 2008, 92, 011911- 011913.

[65] M. Kundu, Y. Murata, Applied Physics Letters 2002, 80, 1921-1923.

[66] Z. Zhang, Z. Jiang, Y. Yao, D. Tan, Q. Fu, X. Bao, Thin Solid Films 2008, 516, 37413746.

[67] D. C. Kershner, J. W. Medlin, Surface Science 2008, 602, 786-794.

[68] D. C. Kershner, W. Zhang, J. Will Medlin, Surface Science 2008, 602, 3225-3231.

[69] D. C. Kershner, M. P. Hyman, J. W. Medlin, Surface Science 2008, 602, 3603-3610.

[70] D. Loeffler, J. J. Uhlrich, M. Baron, B. Yang, X. Yu, L. Lichtenstein, L. Heinke, C. Buechner, M. Heyde, S. Shaikhutdinov, H. J. Freund, R. Wlodarczyk, M. Sierka, J. Sauer, Physical Review Letters 2010, 105, 146104.

[71] a) D. R. Mullins, P. V. Radulovic, S. H. Overbury, Surface Science 1999, 429, 186-198;

b) J. L. Lu, H. J. Gao, S. Shaikhutdinov, H. J. Freund, Surface Science 2006, 600, 50045010. 
[72] B. Yang, W. E. Kaden, X. Yu, J. A. Boscoboinik, Y. Martynova, L. Lichtenstein, M. Heyde, M. Sterrer, R. Wlodarczyk, M. Sierka, J. Sauer, S. Shaikhutdinov, H.-J. Freund, Physical Chemistry Chemical Physics 2012.

[73] R. Włodarczyk, M. Sierka, J. Sauer, D. Löffler, J. J. Uhlrich, X. Yu, B. Yang, I. M. N. Groot, S. Shaikhutdinov, H. J. Freund, Physical Review B 2012, 85, 085403.

[74] a) U. Martinez, L. Giordano, G. Pacchioni, The Journal of Chemical Physics 2008, 128, 164707-164708; b) S. Ulrich, N. Nilius, H.-J. Freund, U. Martinez, L. Giordano, G. Pacchioni, Physical Review Letters 2009, 102, 016102.

[75] X. Yu, B. Yang, J. A. Boscoboinik, S. Shaikhutdinov, H.-J. Freund, Applied Physics Letters 2012, 100, 151608-151604.

[76] W. H. Zachariasen, Journal of the American Chemical Society 1932, 54, 3841-3851.

[77] B. E. Warren, Journal of the American Ceramic Society 1934, 17, 249-254.

[78] R. J. Bell, P. Dean, Philosophical Magazine 1972, 25, 1381-1398.

[79] E. Görlich, K. Błaszczak, G. Sieminska, Journal of Materials Science 1974, 9, 19261932.

[80] L. Lichtenstein, C. Büchner, B. Yang, S. Shaikhutdinov, M. Heyde, M. Sierka, R. Włodarczyk, J. Sauer, H.-J. Freund, Angewandte Chemie International Edition 2012, 51, 404-407.

[81] L. F. Gladden, T. A. Carpenter, S. R. Elliott, Philosophical Magazine Part B 1986, 53, L81-L87.

[82] R. L. Mozzi, B. E. Warren, Journal of Applied Crystallography 1969, 2, 164-172.

[83] L. F. Gladden, Journal of Non-Crystalline Solids 1990, 119, 318-330.

[84] L. Lichtenstein, M. Heyde, H. J. Freund, Phys. Rev. Lett. 2012, 109, 10601 
[85] P. Y. Huang, S. Kurasch, A. Srivastava, V. Skakalova, J. Kotakoski, A. V. Krasheninnikov, R. Hovden, Q. Mao, J. C. Meyer, J. Smet, D. A. Muller, U. Kaiser, Nano Letters 2012, 12, 1081-1086.

[86] J. A. Boscoboinik, X. Yu, B. Yang, F. D. Fischer, R. Włodarczyk, M. Sierka, S.

Shaikhutdinov, J. Sauer, H.-J. Freund, Angewandte Chemie International Edition 2012, $51,6005-6008$. 